\title{
Occupation, industry, and the risk of prostate cancer: a case-control study in Montréal, Canada
}

\author{
Jean-François Sauvé ${ }^{1,2}$, Jérôme Lavoué $e^{1,2}$ and Marie-Élise Parent ${ }^{2,3,4^{*}}$
}

\begin{abstract}
Background: Age, family history and ancestry are the only recognized risk factors for prostate cancer (PCa) but a role for environmental factors is suspected. Due to the lack of knowledge on the etiological factors for PCa, studies that are both hypothesis-generating and confirmatory are still needed. This study explores relationships between employment, by occupation and industry, and PCa risk.

Methods: Cases were 1937 men aged $\leq 75$ years with incident PCa diagnosed across Montreal French hospitals in 2005-2009. Controls were 1994 men recruited concurrently from electoral lists of French-speaking Montreal residents, frequency-matched to cases by age. In-person interviews elicited occupational histories. Unconditional logistic regression estimated odds ratios (OR) and $95 \%$ confidence intervals (Cl) for the association between employment across 696 occupations and 613 industries and PCa risk, adjusting for potential confounders. Multinomial logistic models assessed risks by PCa grade. Semi-Bayes (SB) adjustment accounted for the large number of associations evaluated.
\end{abstract}

Results: Consistently positive associations — and generally robust to SB adjustment-were found for occupations in forestry and logging (OR 1.9, $95 \%$ Cl: 1.2-3.0), social sciences (OR 1.6, $95 \%$ Cl: 1.1-2.2) and for police officers and detectives (OR: 1.8, $95 \% \mathrm{Cl}$ 1.1-2.9). Occupations where elevated risk of high grade PCa was found included gasoline station attendants (OR 4.3, $95 \% \mathrm{Cl} 1.8-10.4)$ and textile processing occupations (OR 1.8, $95 \% \mathrm{Cl}$ 1.1-3.2). Aside from logging, industries with elevated PCa risk included provincial government and financial institutions. Occupations with reduced risk included farmers (OR 0.6, $95 \% \mathrm{Cl}$ 0.4-1.0) and aircraft maintenance workers (OR 0.1, $95 \% \mathrm{Cl} 0.0-0.7$ ).

Conclusions: Excess PCa risks were observed across several occupations, including predominantly white collar workers. Further analyses will focus on specific occupational exposures.

Keywords: Prostate cancer, Occupation, Industry

\section{Background}

Prostate cancer $(\mathrm{PCa})$ is the most frequently diagnosed cancer among Canadian men, with over 20,000 new cases and 4000 deaths per year [1]. PCa is also the most frequently diagnosed cancer in the United States [2] and the second worldwide after lung cancer [3]. Recognized risk factors for PCa are limited to age, first-degree family

\footnotetext{
* Correspondence: marie-elise.parent@iaf.inrs.ca

${ }^{2}$ Centre de recherche du CHUM, 850 rue Saint-Denis, Montréal, Québec H2X OA9, Canada

${ }^{3}$ Epidemiology and Biostatistics UnitEpidemiology and Biostatistics Unit, INRS-Institut Armand-Frappier, Institut national de la recherche scientifique, Université du Québec, 531 Boul. des Prairies, Laval, Québec H7V 1B7, Canada Full list of author information is available at the end of the article
}

history of PCa and ethnicity [4]. The influence of environmental and lifestyle factors in the etiology of this disease has long been suggested, including factors from the work environment, the latter having been reviewed by Parent and Siemiatycki [5] and more recently by Doolan, et al. [6]. Notably, the International Agency for Research on Cancer (IARC) considers that limited evidence exists for arsenic and inorganic compounds, cadmium and cadmium compounds, malathion and $\mathrm{X}$ and gamma radiation, as well as employment in rubber manufacturing [7].

Many studies have focused on farming and pesticide application as elevated $\mathrm{PCa}$ incidence and mortality 
among farmers have been documented since the 1980s. Studies such as the Agricultural Health Study (AHS) in the United States have provided some clues as to agents that might be implicated, mainly organochlorines and organophosphates. More research will help characterize the potential interplay between these exposures and genetic factors in $\mathrm{PCa}$ etiology [8]. Other occupations that have been associated with elevated risks of $\mathrm{PCa}$ include firefighters [9], aviation-related [10, 11], administrative and managerial [12, 13], metalworking [5] and rubber production [14] occupations. However, results from occupation-based studies have generally been inconsistent or inconclusive. Very few of them have taken into account PCa aggressiveness [15-18]. For instance, preliminary results from an American case-control study [18] found relationships between employment in truck driving or gardening occupations and aggressive $\mathrm{PCa}$ relative to lower PCa grade. Associations between aggressive PCa and selected organochlorine and organophosphate pesticides were also found in the AHS [19] which, in the case of Diazinon, was not apparent when looking at total PCa [20].

This study aims at further exploring potential associations between employment across a wide range of occupations and industries and PCa risk, both overall and stratified by $\mathrm{PCa}$ grade, using data from a large, population-based case-control study conducted in Montreal, Canada.

\section{Methods}

\section{Study population}

The Prostate Cancer and Environment Study (PROtEuS) is a population-based case-control study conducted in Greater Montreal, Canada, initiated to explore the role of lifestyle, environmental and occupational factors in PCa etiology. The study has been described in detail previously [21]. Briefly, eligible subjects were men aged $\leq 75$ years at diagnosis or recruitment, Canadian citizens, registered on the permanent electoral list and residing in one of the 39 electoral districts of the greater Montreal area.

Cases were all patients newly diagnosed with primary histologically confirmed PCa from September 2005 to December 2009, actively ascertained from pathology departments across the main Montreal hospitals serving the French-speaking population. These represent over $80 \%$ of all new cases in the area according to registry information. Concurrently, population controls were randomly selected from the electoral list of French-speaking men, frequency-matched to cases by 5 -year age intervals. Participation rates for eligible cases and controls were $79.4 \%$ and $55.5 \%$, respectively. Ethics boards of all participating institutions approved the study; all subjects provided written informed consent.

\section{Data collection}

Between 2006 and 2012, in-person interviews were conducted by trained interviewers, mainly at the subjects' homes, to collect a complete occupational history covering each job held for at least 1 year during their career, including first and last year of employment, company name and main tasks performed. Subjects also provided information on a variety of socio-demographic characteristics, anthropometric, lifestyle and environmental factors, as well as medical and residential histories. Gleason scores were extracted from cases' biopsy pathology reports to define PCa grades.

\section{Coding of occupation and industry titles}

A team of industrial hygienists reviewed the occupational histories (blind to case/control status) to assign occupation and industry titles for each job. Occupations were coded in the 1971 (updated through 1980) Canadian Classification and Dictionary of Occupations (CCDO) [22] scheme, which has a hierarchical structure featuring 2-digit major groups, 3-digit minor groups, 4-digit unit groups and 7digit occupations. Industry titles were based on the 1980 Canadian Standardized Industrial Classification (SIC) [23] defined by 2-digit major groups, 3-digit minor groups and 4-digit industry classes.

\section{Statistical analyses}

For each occupation and industry category, unconditional logistic regression models estimated odds ratios (OR) and $95 \%$ confidence intervals $(95 \% \mathrm{CI})$ for the risk of $\mathrm{PCa}$ according to ever employment and duration of employment ( $<10$ and $\geq 10$ years). For a given occupation or industry category, subjects never employed in that particular occupation or industry represented the reference group. ORs were estimated for each 2, 3, 4 and 7-digit CCDO and 2, 3 and 4-digit SIC categories with at least 10 subjects (including 1 case and 1 control) ever employed.

Models were adjusted for the three recognized risk factors for PCa: age (as a continuous variable), first-degree family history of PCa (yes, no, unknown), ancestry (European, Sub-Saharan African, Asian, Middle Eastern, other (e.g. Hispanic, Aboriginal), unknown) and timing of last PCa screening before diagnosis or interview ( $\leq 2$ years, $>2$ years, never screened, unknown). Inclusion of the following additional covariates in the models was tested using a stepwise procedure and Akaike's Information criterion: Annual household income $(<\$ C 30,000$, \$C30,000$79,999, \$ C 80,000$ or more, refusal, unknown), highest level of education attained (primary, secondary/college, university, unknown), self-reported level of physical activity at work and at home (not very active, moderately active, very active), cigarette pack-years (zero, tertiles above zero, unknown), alcohol drink-years (zero, tertiles above zero, 
unknown) and quartiles of body-mass index (BMI) 2 years before diagnosis or interview. All variables except cigarette pack-years were retained in the final models.

In addition, multinomial logistic regression models were used to evaluate associations according to $\mathrm{PCa}$ grade. Low grade was defined by a Gleason score less than 7 or $(3+4)$ and high grade by a Gleason score of 8 or higher or $(4+3)$ [24]; eight cases were excluded from this analysis due to incomplete information on Gleason scores. The same set of potential confounders retained in the binary logistic regression models was used.

\section{Semi-Bayes adjustment for multiple comparisons}

The large number of occupations and industries evaluated may lead to a non-negligible amount of false-positive associations being observed due to chance. To identify the more robust estimates, we applied a shrinkage-based method using Semi-Bayes adjustment (SB) [25]. Prior applications of SB methods in job title analyses have included separate adjustment for a priori "low" or "high" risk occupations for bladder cancer [26], or accounting for exposures to known lung carcinogens within occupations [27]. In our case, there was no clear a priori expectation of occupations or industries being strongly associated with increased or decreased PCa risk. Therefore, for each combination of cancer grade (all PCa, low or high grade), type of analysis (occupations or industries), and duration (ever/ never or duration in years), estimates were shrunk towards a common mean. For each analysis, a prior variance of 0.25 of the ORs on the log scale was used, corresponding to approximately $95 \%$ of the "true" ORs lying between 0.38 and 2.66 (7-fold difference). In addition, while the analysis was restricted to occupations or industries with at least 10 subjects ever employed, the stratification by duration of employment and by cancer grade could result in estimates based on a smaller sample. Therefore, the application of SB adjustment was limited to categories with at least five subjects employed.

Analyses were performed using the $\mathrm{R}$ software (version 3.1.0, R Development Core Team, Vienna, Austria).

\section{Results}

The study population comprised $1937 \mathrm{PCa}$ cases and 1994 population controls. Among cases, 524 were classified as having high grade $\mathrm{PCa}, 1405$ low grade and 8 had insufficient information to be classified in either category. Proxy respondents, mainly spouses, completed the interview for 50 cases $(3.1 \%)$ and 77 controls (3.9\%). Selected characteristics of cases and controls are presented in Table 1. Controls were slightly older than cases on average at interview (mean of 64.8 years) compared to cases at diagnosis (mean of 63.6 years), reflecting the slightly longer time required to secure interviews with controls. As expected, subjects from Sub-Saharan ancestry and with first-degree family history of PCa were more likely to be cases, while subjects of Asian ancestry were more likely to be controls. The proportion of controls screened at least once by prostatic specific antigen (PSA) and/or digital rectal examination (DRE) in the 2 years preceding the date of interview was relatively high at $76 \%$. Annual household income, highest level of education, physical activity level, cigarette pack-years and alcohol intake level were fairly similar between cases and controls. There were more controls in the highest quartile of BMI compared to cases. The occupational histories of the 3931 subjects covered 19,373 unique jobs spanning the period 1943-2012. The number of jobs held during lifetime per subject ranged from 0 ( 2 cases and 2 controls) to 23, with an average of 4.9 .

A total of 2993 2, 3, 4 and 7-digit CCDO groups were represented in data, with 696 (23\%) having at least 10 subjects ever employed. The risk of PCa (all PCa and by $\mathrm{PCa}$ grade) associated with each of these 696 groups are presented in [Additional file 1: Table S1], while remaining occupations $(n=2297)$ are listed in [Additional file 2 Table S3]. Occupations with a significantly elevated or reduced PCa risk are presented in Table 2 for all PCa, and in Table 3 for high grade $\mathrm{PCa}$.

Two 2-digit categories were associated with statistically elevated ORs for ever employment in the following groups: Occupations in social sciences (OR 1.6, 95 \% CI: 1.1-2.2) and Forestry and logging occupations (OR 1.9, 95 \% CI: 1.2-3.0). Subgroups with increased PCa risk within these categories include educational counsellors (OR 3.0, 95 \% CI 1.1-8.3) and loggers (OR 2.0, 95 \% CI 1.2-3.4). Elevated ORs were also found for ever employment as Police officers and detectives (OR 1.8, $95 \% \mathrm{CI}$ 1.1-2.9), Mixing and blending occupations (OR 3.6, $95 \%$ CI 1.2-10.8), Governmental inspectors and regulatory officers (OR 2.7, 95 \% CI 1.2-6.2), employment $<10$ years in Painting and decorating occupations excepting construction (OR 3.0, 95 \% CI 1.3-7.0), and in occupations associated with administration and sales, such as Receptionists, General office clerks for ever and $<10$ years employed, and Commodities sales clerks employed $<10$ years.

Associations for high grade $\mathrm{PCa}$, estimated using multinomial models, were observed for ever employment in Social sciences occupations (OR 1.8, 95 \% CI 1.1-3.0) and Forestry and logging (OR 2.5, 95 \% CI 1.4-4.5). For the latter, the association was the strongest for employment $\geq 10$ years (OR 4.4, 95 \% CI 1.5-12.7). Other groups where associations were mainly limited to high grade PCa were Service station attendants (OR ever 4.3, 95 \% CI 1.8-10.4), Textile processing occupations (OR ever 1.8, 95 \% CI 1.13.2), and Bus drivers (OR <10 years 2.9, $95 \%$ CI 1.1-7.3).

Regarding decreased PCa risk, negative associations were found for employment in Farming; specific occupations 
Table 1 Selected characteristics of cases and controls

\begin{tabular}{|c|c|c|c|c|}
\hline \multirow[t]{2}{*}{ Variable } & \multirow{2}{*}{$\begin{array}{l}\text { Controls } \\
(n=1994)\end{array}$} & \multicolumn{3}{|l|}{ Cases } \\
\hline & & All $(n=1937)$ & Low grade $^{a}(n=1405)$ & High grade $^{\mathrm{b}}(n=524)$ \\
\hline \multicolumn{5}{|c|}{ Age at diagnosis or interview in years, $\mathrm{n}(\%)$} \\
\hline$<60$ & $446(22.4)$ & $523(27.0)$ & $414(29.5)$ & $107(20.4)$ \\
\hline$\geq 60$ and $<65$ & $450(22.6)$ & $486(25.1)$ & $351(25.0)$ & $131(25.0)$ \\
\hline$\geq 65$ and $<70$ & $522(26.2)$ & $498(25.7)$ & $347(24.7)$ & $150(28.6)$ \\
\hline$\geq 70$ and $\leq 75$ & $576(28.9)$ & $430(22.2)$ & $293(20.9)$ & $136(26.0)$ \\
\hline \multicolumn{5}{|l|}{ Ancestry, n (\%) } \\
\hline European & $1685(84.5)$ & $1696(87.6)$ & $1234(87.8)$ & $455(86.8)$ \\
\hline Sub-Saharan & $90(4.5)$ & $130(6.7)$ & $99(7.0)$ & $30(5.7)$ \\
\hline Asian & $73(3.7)$ & $24(1.2)$ & $15(1.1)$ & $9(1.7)$ \\
\hline Greater Middle East & $99(5.0)$ & $45(2.3)$ & $32(2.3)$ & $13(2.5)$ \\
\hline Other & $33(1.7)$ & $30(1.5)$ & $18(1.3)$ & $12(2.3)$ \\
\hline Don't know & $14(0.7)$ & $12(0.6)$ & $7(0.5)$ & $5(1.0)$ \\
\hline \multicolumn{5}{|c|}{ First-degree family history of PCa, n (\%) } \\
\hline No & $1739(87.2)$ & $1419(73.3)$ & $1008(71.7)$ & $405(77.3)$ \\
\hline Yes & $199(10.0)$ & $452(23.3)$ & $351(25.0)$ & $100(19.1)$ \\
\hline Don't know & $56(2.8)$ & $66(3.4)$ & $46(3.3)$ & $19(3.6)$ \\
\hline \multicolumn{5}{|c|}{ Date of last screening for PCa before diagnosis or interview, n (\%) } \\
\hline$\leq 2$ years & $1510(75.7)$ & $1917(99.0)$ & $1388(98.8)$ & $521(99.4)$ \\
\hline$>2$ years & $235(11.8)$ & $1(0.1)$ & $1(0.1)$ & $0(0.0)$ \\
\hline No/Never & $191(9.6)$ & $3(0.2)$ & $2(0.1)$ & $1(0.2)$ \\
\hline Don't know & $58(2.9)$ & $16(0.8)$ & $14(1.0)$ & $2(0.4)$ \\
\hline \multicolumn{5}{|c|}{ Annual household income in CAD\$, n (\%) } \\
\hline$<10,000-29,999$ & $497(24.9)$ & $490(25.3)$ & $323(23.0)$ & $167(31.9)$ \\
\hline $30,000-79,999$ & $872(43.8)$ & $874(45.1)$ & $640(45.6)$ & $228(43.5)$ \\
\hline 80,000 and more & $428(21.5)$ & $426(22.0)$ & $343(24.4)$ & $81(15.5)$ \\
\hline Prefers not to respond & $186(9.3)$ & $132(6.8)$ & $91(6.5)$ & $41(7.8)$ \\
\hline Don't know & $9(0.5)$ & $15(0.8)$ & $8(0.6)$ & $7(1.3)$ \\
\hline \multicolumn{5}{|c|}{ Highest level of education attained, n (\%) } \\
\hline Primary & $428(21.5)$ & $449(23.2)$ & $312(22.2)$ & $136(26.0)$ \\
\hline Secondary/College & $953(47.8)$ & $891(46.0)$ & $629(44.8)$ & $259(49.5)$ \\
\hline University & $611(30.7)$ & $592(30.6)$ & $461(32.8)$ & $127(24.3)$ \\
\hline Don't know & $1(0.1)$ & $4(0.2)$ & $3(0.2)$ & $1(0.2)$ \\
\hline \multicolumn{5}{|c|}{ Self-reported level of physical activity at work or leisure, n (\%) } \\
\hline Not very active & $151(7.6)$ & $127(6.6)$ & $84(6.0)$ & $43(8.2)$ \\
\hline Moderately active & $753(37.8)$ & $682(35.2)$ & $516(36.7)$ & $160(30.5)$ \\
\hline Very active & $1089(54.6)$ & $1128(58.2)$ & $805(57.3)$ & $321(61.3)$ \\
\hline \multicolumn{5}{|l|}{ Cigarette pack-years, n (\%) } \\
\hline Zero & $544(27.3)$ & $542(28.0)$ & $404(28.8)$ & $136(26.0)$ \\
\hline$>0-15.1$ & $441(22.1)$ & $498(25.7)$ & $381(27.1)$ & $114(21.8)$ \\
\hline$>15.1-39.4$ & $524(26.3)$ & $420(21.7)$ & $296(21.1)$ & $122(23.3)$ \\
\hline$>39.4-223$ & $474(23.8)$ & $458(23.6)$ & $313(22.3)$ & $144(27.5)$ \\
\hline Don't know & $11(0.6)$ & $19(1.0)$ & $11(0.8)$ & $8(1.5)$ \\
\hline
\end{tabular}


Table 1 Selected characteristics of cases and controls (Continued)

\begin{tabular}{|c|c|c|c|c|}
\hline \multicolumn{5}{|c|}{ Alcohol drink-years, n (\%) } \\
\hline Zero & $231(11.6)$ & $214(11.0)$ & $157(11.2)$ & $56(10.7)$ \\
\hline$>0-24.4$ & $575(28.8)$ & $548(28.3)$ & $413(29.4)$ & $133(25.4)$ \\
\hline$>24.4-76$ & $569(28.5)$ & $553(28.5)$ & $408(29.0)$ & $143(27.3)$ \\
\hline$>76-2660$ & $577(28.9)$ & $545(28.1)$ & $376(26.8)$ & $166(31.7)$ \\
\hline Don't know & $42(2.1)$ & $77(4.0)$ & $51(3.6)$ & $26(5.0)$ \\
\hline \multicolumn{5}{|c|}{ Body-mass index, 2 years before diagnosis or interview, in $\mathrm{kg} / \mathrm{m}^{2}, \mathrm{n}(\%)$} \\
\hline$\leq 24.2$ & $477(23.9)$ & $502(25.9)$ & $362(25.8)$ & $137(26.2)$ \\
\hline$>24.2-26.5$ & $492(24.7)$ & $512(26.4)$ & $373(26.5)$ & $137(26.2)$ \\
\hline$>26.5-29.2$ & 477 (23.9) & $479(24.7)$ & $362(25.8)$ & $116(22.2)$ \\
\hline$>29.2$ & $535(26.8)$ & $431(22.3)$ & $299(21.3)$ & $130(24.9)$ \\
\hline Don't know & $13(0.7)$ & $12(0.6)$ & $9(0.6)$ & $3(0.6)$ \\
\hline
\end{tabular}

${ }^{\mathrm{a}}$ Gleason score $\leq 6$ or $3+4$

${ }^{\mathrm{b}}$ Gleason score $\geq 8$ or $4+3$

within farming where such associations were observed were General farmers (OR $<10$ years $0.3,95 \% \mathrm{CI} 0.1-0.9$ ), and Field crop and Vegetable growing workers (OR ever 0.3 , $95 \%$ CI 0.1-0.9). Decreased PCa risk was also found in occupations associated with Aircraft and Air transportation, and for ever and $<10$ years employment in Electrical engineers.

Applying SB adjustment for the analysis by occupation led to an attenuation of risk estimates, especially in occupations with few subjects ever employed. For example, the number of categories with a statistically significant association between ever employment and PCa risk saw a threefold reduction, from 37 to 12 . The more robust associations for total PCa risk were found for ever employment in Occupations in social sciences (ORsb 1.5, $95 \%$ CI 1.1-2.1), Police officers (ORsb 1.7, 95 \% CI 1.0-2.7) and Forestry and logging occupations (ORsb $1.7,95 \%$ CI 1.1-2.6). For high grade PCa, these included Occupations in social sciences, Forestry and logging, Bookkeeping clerks and Service station attendants. Decreased PCa risk associated with employment $<10$ years as Farmer also remained robust to shrinkage (ORsb 0.5, $95 \%$ CI 0.3-0.9).

For the analysis by industry title, the number of 2,3 and 4-digit industry categories with at least one subject ever employed totaled 1125, with 613 (54\%) featuring at least 10 subjects ever employed. Associations between PCa risk and employment in these 613 categories are presented in [Additional file 3: Table S2]; categories with at least one statistically significant association for total PCa are presented in Tables 4 and 5 for high grade PCa. The remaining 512 industry groups excluded from the analysis are listed in [Additional file 2: Table S3].

Some industries with elevated PCa risk, such as Logging industry and Protective services, provincial reflect the results from the corresponding categories for occupations, i.e. Forestry and logging occupations and Police officers and detectives, respectively. Other major industries where elevated PCa risk was found include Provincial and territorial governments (OR ever 1.5, $95 \%$ CI 1.1-2.0) and Finance, such as banks and investment intermediaries. Positive associations were also found for employment $<10$ years in Urban transit systems (OR 2.4, 95 \% CI 1.05.8 ) and $\geq 10$ years in the Paper products industry (OR 2.3, $95 \%$ CI 1.1-5.0). Elevated risk for high grade PCa was found for Provincial and territorial governments industry (OR ever 1.7, 95 \% CI 1.2-2.6); in addition, elevated risk was found for employment $<10$ years in Local government service (OR 1.9, 95 \% CI 1.1-3.4). Other industries with increased risk of high grade $\mathrm{PCa}$ include ever employment in Wood industries (OR 1.9, 95 \% CI 1.0-3.6), Primary steel industries (OR 2.1, $95 \%$ CI 1.0-4.5) and Gasoline service stations (OR 2.8, $95 \%$ CI 1.4-5.5), as well as employment $\geq 10$ years in Truck transportation (OR 2.0, $95 \%$ CI 1.1-3.5)

Lower PCa risk was found for ever employment in Agriculture (OR 0.6, 95 \% CI 0.4-0.9); Livestock combination farms was the only nested industry with a statistically significantly reduced association. Employments $\geq 10$ years in the Aircraft (OR 0.5, $95 \%$ CI 0.-0.8) and Air transportation (OR $0.4,95 \% 0.2-0.9$ ) industries were associated with lower PCa risk. Employment in Food and beverage service, including restaurants, was also inversely associated with PCa.

Industries where significant positive associations with all PCa remained after SB adjustment included Logging, Chartered banks, Investment intermediaries and Provincial and territorial governments. Aside from Provincial government and Logging industries, robust associations for high grade $\mathrm{PCa}$ included employment $\geq 10$ years in Truck transport (ORsb 1.7, $95 \%$ CI 1.1-2.8) and employment $<10$ years for Gasoline service stations (ORsb 
Table 2 Associations between selected occupations and risk of prostate cancer (all cancers) ${ }^{\text {a }}$

\begin{tabular}{|c|c|c|c|c|c|c|c|c|c|c|}
\hline \multirow[t]{2}{*}{ CCDO code and description } & \multirow{2}{*}{$\begin{array}{l}\text { Never } \\
\mathrm{Ca} / \mathrm{Co}\end{array}$} & \multicolumn{3}{|c|}{ Ever employed } & \multicolumn{3}{|c|}{$<10$ years employed } & \multicolumn{3}{|c|}{$\geq 10$ years employed } \\
\hline & & $\mathrm{Ca} / \mathrm{Co}$ & OR $(95 \% \mathrm{Cl})$ & OR SB (95\% Cl) & $\mathrm{Ca} / \mathrm{Co}$ & OR $(95 \% \mathrm{Cl})$ & OR SB (95\% Cl) & $\mathrm{Ca} / \mathrm{Co}$ & OR $(95 \% \mathrm{Cl})$ & OR SB $(95 \% \mathrm{Cl})$ \\
\hline 111: Officials and administrators unique to government & $1875 / 1936$ & $62 / 58$ & $1.2(0.8-1.8)$ & $1.2(0.8-1.7)$ & $19 / 31$ & $0.7(0.4-1.3)$ & $0.8(0.5-1.3)$ & $43 / 27$ & $1.8(1.0-3.1)^{\mathrm{b}}$ & $1.6(0.9-2.5)$ \\
\hline 1116: Inspectors and regulatory officers, government & 1910/1982 & $27 / 12$ & $2.7(1.2-6.2)^{b}$ & $1.8(1.0-3.4)$ & $11 / 4$ & $2.9(0.8-10.7)$ & $1.5(0.7-3.3)$ & $16 / 8$ & $2.6(0.9-7.4)$ & $1.6(0.8-3.2)$ \\
\hline 1130126: General manager, finance (bank. \& finance) & 1925/1989 & $12 / 5$ & $3.5(1.0-12.5)^{\mathrm{b}}$ & $1.6(0.8-3.5)$ & $5 / 3$ & $1.9(0.4-10.2)$ & $1.2(0.5-2.8)$ & $7 / 2$ & $6.7(0.9-48.2)$ & $1.5(0.6-3.5)$ \\
\hline 1143: Production management occupations & $1911 / 1954$ & $26 / 40$ & $0.7(0.4-1.3)$ & $0.8(0.5-1.3)$ & $12 / 12$ & $1.6(0.6-4.0)$ & $1.3(0.7-2.5)$ & $14 / 28$ & $0.5(0.2-1.0)^{b}$ & $0.6(0.4-1.1)$ \\
\hline 1171162: Auditor (prof. \& tech., n.e.c.) & 1908/1975 & 29/19 & $1.7(0.9-3.3)$ & $1.4(0.8-2.5)$ & $9 / 11$ & $0.9(0.3-2.3)$ & $0.9(0.5-1.9)$ & $20 / 8$ & $2.9(1.1-7.3)^{b}$ & $1.8(0.9-3.4)$ \\
\hline $\begin{array}{l}\text { 1179299: Other occupations related to management } \\
\text { and administration }\end{array}$ & 1916/1985 & $21 / 9$ & $2.8(1.1-6.9)^{\mathrm{b}}$ & $1.7(0.9-3.4)$ & $12 / 6$ & $2.5(0.8-7.7)$ & $1.5(0.7-3.1)$ & $9 / 3$ & $3.4(0.7-16.3)$ & $1.4(0.6-3.3)$ \\
\hline $\begin{array}{l}\text { 21: Occupations in natural sciences, engineering and } \\
\text { mathematics }\end{array}$ & $1713 / 1722$ & $224 / 272$ & $0.8(0.7-1.1)$ & $0.8(0.7-1.1)$ & $85 / 75$ & $1.1(0.8-1.6)$ & $1.1(0.8-1.6)$ & 139/197 & $0.7(0.6-0.9)^{b}$ & $0.8(0.6-1.0)^{\mathrm{b}}$ \\
\hline 2144: Electrical engineers & 1926/1965 & $11 / 29$ & $0.4(0.2-0.8)^{b}$ & $0.5(0.3-1.0)^{b}$ & $2 / 13$ & $0.1(0.0-0.5)^{b}$ & $0.5(0.2-1.2)$ & 9/16 & $0.8(0.3-1.9)$ & $0.9(0.4-1.7)$ \\
\hline $\begin{array}{l}\text { 2144110: Design and development engineer, } \\
\text { electrical and electronic (prof. \& tech., n.e.c.) }\end{array}$ & 1934/1987 & $3 / 7$ & $0.2(0.1-1.0)$ & $0.6(0.3-1.5)$ & $1 / 5$ & $0.1(0.0-0.8)^{b}$ & $0.7(0.3-1.6)$ & $2 / 2$ & $1.5(0.1-17.6)$ & \\
\hline 23: Occupations in social sciences and related fields & $1809 / 1914$ & $128 / 80$ & $1.6(1.1-2.2)^{b}$ & $1.5(1.1-2.1)^{b}$ & $52 / 30$ & $1.6(1.0-2.6)$ & $1.4(0.9-2.3)$ & $76 / 50$ & $1.6(1.0-2.5)^{b}$ & $1.5(1.0-2.2)^{b}$ \\
\hline 2391118: Counsellor, educational (educ.) & 1918/1988 & $19 / 6$ & $3.0(1.1-8.3)^{b}$ & $1.7(0.9-3.5)$ & $12 / 4$ & $2.4(0.8-7.4)$ & $1.4(0.7-3.0)$ & $7 / 2$ & $5.7(0.8-43.4)$ & $1.4(0.6-3.4)$ \\
\hline 271: University teaching and related occupations & 1852/1933 & $85 / 61$ & $1.5(1.0-2.2)^{b}$ & $1.4(1.0-2.0)$ & $29 / 20$ & $1.4(0.7-2.6)$ & $1.3(0.7-2.2)$ & $56 / 41$ & $1.5(1.0-2.4)$ & $1.4(0.9-2.2)$ \\
\hline 315: Other occupations in medicine and health & 1913/1973 & $24 / 21$ & $1.0(0.5-1.9)$ & $1.0(0.6-1.7)$ & $19 / 6$ & $3.2(1.0-9.8)^{b}$ & $1.7(0.8-3.5)$ & $5 / 15$ & $0.3(0.1-0.9)^{b}$ & $0.6(0.3-1.2)$ \\
\hline 335: Occupations in writing & 1919/1962 & $18 / 32$ & $0.6(0.3-1.2)$ & $0.7(0.4-1.2)$ & $10 / 12$ & $1.5(0.5-4.3)$ & $1.2(0.6-2.5)$ & $8 / 20$ & $0.3(0.1-0.8)^{b}$ & $0.6(0.3-1.0)$ \\
\hline 4171: Receptionists and information clerks & 1918/1986 & $19 / 8$ & $3.0(1.1-8.0)^{b}$ & $1.7(0.9-3.5)$ & $19 / 8$ & $3.0(1.1-8.0)^{\mathrm{b}}$ & $1.7(0.9-3.5)$ & $0 / 0$ & & \\
\hline 4197: General office clerks & $1839 / 1910$ & $98 / 84$ & $1.3(0.9-1.8)$ & $1.2(0.9-1.7)$ & $81 / 53$ & $1.6(1.1-2.3)^{b}$ & $1.5(1.0-2.1)^{b}$ & $17 / 31$ & $0.6(0.3-1.2)$ & $0.7(0.4-1.3)$ \\
\hline 513/514: Sales occupations, commodities & $1564 / 1569$ & $373 / 425$ & $0.9(0.7-1.0)$ & $0.9(0.7-1.0)$ & 198/193 & $1.0(0.8-1.3)$ & $1.0(0.8-1.3)$ & $175 / 232$ & $0.8(0.6-0.9)^{b}$ & $0.8(0.6-0.9)^{b}$ \\
\hline 5135182: Salesperson, footwear (ret. Trade) & 1936/1985 & $1 / 9$ & $0.1(0.0-0.9)^{b}$ & $0.7(0.3-1.7)$ & $0 / 7$ & & & $1 / 2$ & $0.8(0.1-8.9)$ & \\
\hline 5137: Sales clerks, commodities & 1833/1908 & $104 / 86$ & $1.2(0.9-1.7)$ & $1.2(0.9-1.6)$ & $82 / 58$ & $1.5(1.0-2.3)^{\mathrm{b}}$ & $1.5(1.0-2.1)^{\mathrm{b}}$ & $22 / 28$ & $0.7(0.4-1.2)$ & $0.8(0.4-1.2)$ \\
\hline 5137111: Supermarket clerk (ret. Trade) & $1900 / 1968$ & $37 / 26$ & $1.6(0.9-2.9)$ & $1.4(0.9-2.4)$ & $32 / 18$ & $2.3(1.2-4.7)^{b}$ & $1.8(1.0-3.1)^{\mathrm{b}}$ & $5 / 8$ & $0.5(0.2-1.7)$ & $0.8(0.4-1.6)$ \\
\hline 5145: Service station attendants & 1912/1982 & $25 / 12$ & $2.3(1.0-5.1)^{b}$ & $1.7(0.9-3.1)$ & $21 / 11$ & $2.0(0.9-4.8)$ & $1.5(0.8-2.9)$ & $4 / 1$ & $4.6(0.5-42.0)$ & $1.3(0.5-3.2)$ \\
\hline 5199158: Telephone solicitor (any ind.) & 1930/1981 & $7 / 13$ & $0.5(0.2-1.3)$ & $0.7(0.3-1.4)$ & $3 / 12$ & $0.2(0.1-0.9)^{b}$ & $0.6(0.3-1.3)$ & $4 / 1$ & $2.7(0.3-24.6)$ & $1.2(0.5-2.9)$ \\
\hline 6112: Police officers and detectives, government & 1892/1964 & $45 / 30$ & $1.8(1.1-2.9)^{b}$ & $1.6(1.0-2.4)$ & $13 / 5$ & $3.2(1.0-10.0)^{b}$ & $1.7(0.8-3.5)$ & $32 / 25$ & $1.5(0.8-2.6)$ & $1.4(0.8-2.2)$ \\
\hline 6112146: Detective (gov. Serv.) & 1925/1987 & $12 / 7$ & $2.0(0.7-5.2)$ & $1.4(0.7-2.8)$ & $9 / 1$ & $10.3(1.3-82.9)^{b}$ & $1.5(0.6-3.8)$ & $3 / 6$ & $0.5(0.1-2.3)$ & $0.8(0.4-1.9)$ \\
\hline 6112158: Police officer (gov. Serv.) & $1899 / 1972$ & $38 / 22$ & $2.0(1.1-3.5)^{b}$ & $1.7(1.0-2.7)^{\mathrm{b}}$ & $18 / 7$ & $3.4(1.3-8.8)^{b}$ & $1.9(0.9-3.7)$ & $20 / 15$ & $1.4(0.7-2.9)$ & $1.3(0.7-2.3)$ \\
\hline 6117190: Infantry soldier (military) & $1877 / 1898$ & $60 / 96$ & $0.7(0.5-1.0)$ & $0.7(0.5-1.0)$ & $57 / 95$ & $0.7(0.5-1.0)^{\mathrm{b}}$ & $0.7(0.5-1.0)$ & $3 / 1$ & $2.4(0.2-24.2)$ & \\
\hline $\begin{array}{l}\text { 612: Food and beverage preparation and related } \\
\text { service occupations }\end{array}$ & $1797 / 1813$ & $140 / 181$ & $0.7(0.6-1.0)^{b}$ & $0.8(0.6-1.0)^{b}$ & $77 / 103$ & $0.7(0.5-1.0)^{\mathrm{b}}$ & $0.7(0.5-1.0)$ & $63 / 78$ & $0.8(0.6-1.2)$ & $0.8(0.6-1.2)$ \\
\hline 6121130: Short-order cook (cater. \& lodg.) & 1933/1982 & $4 / 12$ & $0.2(0.1-0.8)^{b}$ & $0.6(0.3-1.3)$ & $2 / 7$ & $0.3(0.0-1.6)$ & $0.8(0.3-1.8)$ & $2 / 5$ & $0.2(0.0-1.2)$ & $0.7(0.3-1.7)$ \\
\hline
\end{tabular}


Table 2 Associations between selected occupations and risk of prostate cancer (all cancers) ${ }^{\text {a }}$ (Continued)

613: Occupations in lodging and other accommodation

6145: Travel and related attendants, except food and beverage

\section{9: Other service occupations}

6191126: Hospital cleaner (misc. Serv.)

6198: Occupations in labouring and other elemental work, services

71: Farming. Horticultural and animal-husbandry

occupation

711: Farmers

7111: General farmers

7111110: Farmer, general (agric.)

7183: Field crop and vegetable-growing workers

7183122: Farm worker, vegetable (agric.)

75: Forestry and logging occupations

751: Forestry and logging occupations

7513: Timber cutting and related occupations

7513122: Logger, all-round (forest. \& log.)

8161: Mixing and blending occupations, chemicals and related materials

8161218: Mixer (chem., n.e.c.; paint \& varn.)

8529: Other fabricating and assembling

occupations, metal products, n.e.c.

858: Mechanics and repairmen, n.e.c.

8581118: Industrial-truck mechanic (mech.

Equip., n.e.c.)

8582: Aircraft mechanics and repairmen

8595: Painting and decorating occupations, except construction

873: Electrical power, lighting and wire communications equipment erecting, installing and repairing occupations

8739: Electrical power, lighting and wire communications equipment erecting, installing and repairing

occupations, n.e.c.

8784: Plasterers and related occupations

\begin{tabular}{|c|c|c|c|c|c|c|c|c|c|}
\hline 1915/1965 & $22 / 29$ & $0.7(0.4-1.2)$ & $0.8(0.4-1.3)$ & $16 / 13$ & $1.1(0.5-2.5)$ & $1.1(0.6-2.0)$ & $6 / 16$ & $0.3(0.1-0.9)^{b}$ & $0.6(0.3-1.2)$ \\
\hline 1935/1986 & $2 / 8$ & $0.2(0.0-0.9)^{b}$ & $0.6(0.3-1.5)$ & $1 / 4$ & $0.2(0.0-2.4)$ & $0.8(0.3-2.0)$ & $1 / 4$ & $0.1(0.0-1.5)$ & $0.8(0.3-1.9)$ \\
\hline 1759/1799 & $178 / 195$ & $0.8(0.6-1.0)^{b}$ & $0.8(0.6-1.0)^{b}$ & $117 / 113$ & $0.8(0.6-1.1)$ & $0.8(0.6-1.1)$ & $61 / 82$ & $0.7(0.5-1.0)$ & $0.7(0.5-1.0)$ \\
\hline 1921/1973 & $16 / 21$ & $0.6(0.3-1.2)$ & $0.7(0.4-1.3)$ & $8 / 15$ & $0.3(0.1-0.9)^{b}$ & $0.6(0.3-1.1)$ & $8 / 6$ & $1.4(0.4-4.6)$ & $1.2(0.6-2.5)$ \\
\hline 1871/1931 & $66 / 63$ & $0.9(0.6-1.3)$ & $0.9(0.6-1.3)$ & $60 / 47$ & $1.0(0.7-1.6)$ & $1.0(0.7-1.6)$ & $6 / 16$ & $0.3(0.1-0.9)^{b}$ & $0.6(0.3-1.2)$ \\
\hline 1840/1860 & $97 / 134$ & $0.7(0.5-1.0)^{b}$ & $0.8(0.6-1.0)$ & $59 / 82$ & $0.7(0.5-1.1)$ & $0.8(0.5-1.1)$ & $38 / 52$ & $0.7(0.4-1.1)$ & $0.8(0.5-1.2)$ \\
\hline 1903/1941 & $34 / 53$ & $0.6(0.4-1.0)^{b}$ & $0.7(0.4-1.0)$ & $14 / 31$ & $0.4(0.2-0.8)^{b}$ & $0.5(0.3-0.9)^{b}$ & $20 / 22$ & $0.9(0.5-1.9)$ & $1.0(0.5-1.7)$ \\
\hline 1923/1974 & $14 / 20$ & $0.6(0.3-1.2)$ & $0.7(0.4-1.3)$ & $5 / 12$ & $0.3(0.1-0.9)^{b}$ & $0.6(0.3-1.2)$ & $9 / 8$ & $1.1(0.4-3.2)$ & $1.0(0.5-2.1)$ \\
\hline 1923/1974 & $14 / 20$ & $0.6(0.3-1.2)$ & $0.7(0.4-1.3)$ & $5 / 12$ & $0.3(0.1-0.9)^{b}$ & $0.6(0.3-1.2)$ & $9 / 8$ & $1.1(0.4-3.2)$ & $1.0(0.5-2.1)$ \\
\hline 1932/1978 & $5 / 16$ & $0.3(0.1-0.9)^{b}$ & $0.6(0.3-1.2)$ & $4 / 11$ & $0.4(0.1-1.4)$ & $0.7(0.3-1.6)$ & $1 / 5$ & $0.2(0.0-1.6)$ & $0.8(0.3-1.9)$ \\
\hline 1935/1983 & $2 / 11$ & $0.2(0.0-0.9)^{b}$ & $0.6(0.3-1.5)$ & $2 / 7$ & $0.4(0.1-2.4)$ & $0.8(0.3-1.9)$ & $0 / 4$ & & \\
\hline 1874/1964 & $63 / 30$ & $1.9(1.2-3.0)^{b}$ & $1.7(1.1-2.6)^{b}$ & $46 / 23$ & $1.7(1.0-3.0)$ & $1.5(0.9-2.5)$ & $17 / 7$ & $2.4(0.9-6.2)$ & $1.6(0.8-3.1)$ \\
\hline $1874 / 1964$ & $63 / 30$ & $1.9(1.2-3.0)^{b}$ & $1.7(1.1-2.6)^{b}$ & $46 / 23$ & $1.7(1.0-3.0)$ & $1.5(0.9-2.5)$ & $17 / 7$ & $2.4(0.9-6.2)$ & $1.6(0.8-3.1)$ \\
\hline 1884/1969 & $53 / 25$ & $1.9(1.1-3.2)^{b}$ & $1.6(1.0-2.6)^{b}$ & $38 / 20$ & $1.7(0.9-3.0)$ & $1.5(0.9-2.4)$ & $15 / 5$ & $2.8(0.9-8.4)$ & $1.6(0.8-3.3)$ \\
\hline 1884/1970 & $53 / 24$ & $2.0(1.2-3.4)^{b}$ & $1.7(1.1-2.7)^{b}$ & $38 / 19$ & $1.8(1.0-3.2)$ & $1.5(0.9-2.5)$ & $15 / 5$ & $2.8(0.9-8.4)$ & $1.6(0.8-3.3)$ \\
\hline 1919/1986 & $18 / 8$ & $3.6(1.2-10.8)^{b}$ & $1.8(0.9-3.7)$ & $12 / 4$ & $8.0(1.3-50.2)^{b}$ & $1.6(0.7-3.8)$ & $6 / 4$ & $1.9(0.4-7.7)$ & $1.2(0.6-2.8)$ \\
\hline 1926/1991 & $11 / 3$ & $8.0(1.2-53.8)^{b}$ & $1.6(0.6-3.7)$ & $8 / 2$ & $6.2(0.8-47.8)$ & $1.4(0.6-3.5)$ & $3 / 1$ & $20.6(0.22 .7)$ & \\
\hline 1922/1986 & $15 / 8$ & $1.8(0.6-4.9)$ & $1.3(0.6-2.7)$ & $8 / 7$ & $0.8(0.2-2.5)$ & $0.9(0.4-1.9)$ & $7 / 1$ & $19.8(1.0 .5)^{b}$ & $1.4(0.5-3.4)$ \\
\hline 1782/1811 & $155 / 183$ & $0.8(0.6-1.1)$ & $0.8(0.6-1.1)$ & $63 / 64$ & $1.1(0.7-1.6)$ & $1.1(0.7-1.6)$ & $92 / 119$ & $0.7(0.5-1.0)^{b}$ & $0.7(0.5-1.0)^{b}$ \\
\hline 1934/1983 & $3 / 11$ & $0.2(0.0-0.8)^{b}$ & $0.6(0.3-1.3)$ & $1 / 4$ & $0.4(0.0-4.2)$ & $0.9(0.4-2.2)$ & $2 / 7$ & $0.1(0.0-0.8)^{b}$ & $0.6(0.3-1.4)$ \\
\hline 1936/1976 & $1 / 18$ & $0.1(0.0-0.7)^{b}$ & $0.6(0.3-1.6)$ & $0 / 6$ & & & $1 / 12$ & $0.1(0.0-1.1)$ & $0.7(0.3-1.7)$ \\
\hline 1902/1975 & $35 / 19$ & $1.9(1.0-3.5)$ & $1.6(0.9-2.6)$ & $27 / 9$ & $3.0(1.3-7.0)^{b}$ & $1.9(1.0-3.6)$ & $8 / 10$ & $0.8(0.3-2.4)$ & $0.9(0.4-1.9)$ \\
\hline 1852/1924 & $85 / 70$ & $1.3(0.9-1.8)$ & $1.2(0.9-1.8)$ & $41 / 28$ & $2.0(1.1-3.6)^{b}$ & $1.7(1.0-2.8)^{\mathrm{b}}$ & $44 / 42$ & $0.9(0.6-1.5)$ & $1.0(0.6-1.5)$ \\
\hline 1933/1980 & $4 / 14$ & $0.3(0.1-0.8)^{b}$ & $0.6(0.3-1.2)$ & $2 / 8$ & $0.2(0.0-1.1)$ & $0.7(0.3-1.6)$ & $2 / 6$ & $0.3(0.1-1.6)$ & $0.7(0.3-1.7)$ \\
\hline 1933/1985 & $4 / 9$ & $0.2(0.1-0.9)^{b}$ & $0.6(0.3-1.3)$ & $0 / 5$ & & & $4 / 4$ & $0.4(0.1-1.8)$ & $0.8(0.3-1.7)$ \\
\hline $1886 / 1954$ & $51 / 40$ & $1.7(1.0-2.8)^{b}$ & $1.5(1.0-2.4)$ & 28/19 & $1.8(0.9-3.5)$ & $1.5(0.8-2.6)$ & $23 / 21$ & $1.6(0.7-3.4)$ & $1.3(0.7-2.4)$ \\
\hline
\end{tabular}

8791: Pipefitting, plumbing and related occupations, n.e.c. 
Table 2 Associations between selected occupations and risk of prostate cancer (all cancers) ${ }^{\mathrm{a}}$ (Continued)

\begin{tabular}{|c|c|c|c|c|c|c|c|c|c|c|}
\hline 8799: Other construction trades occupations, n.e.c. & $1877 / 1948$ & $60 / 46$ & $1.8(1.1-2.9)^{b}$ & $1.6(1.0-2.5)^{b}$ & $36 / 26$ & $1.6(0.9-3.0)$ & $1.4(0.8-2.4)$ & $24 / 20$ & $2.1(0.9-4.6)$ & $1.6(0.8-2.9)$ \\
\hline 911: Air transport operating operations & 1922/1974 & $15 / 20$ & $0.6(0.3-1.3)$ & $0.7(0.4-1.3)$ & $11 / 9$ & $1.3(0.5-3.6)$ & $1.2(0.6-2.3)$ & $4 / 11$ & $0.2(0.1-0.7)^{b}$ & $0.6(0.3-1.2)$ \\
\hline 915: Water transport operating occupations & 1923/1971 & $14 / 23$ & $0.6(0.3-1.2)$ & $0.7(0.4-1.3)$ & $7 / 18$ & $0.3(0.1-0.8)^{b}$ & $0.6(0.3-1.1)$ & $7 / 5$ & $1.7(0.5-6.3)$ & $1.2(0.6-2.7)$ \\
\hline 9173: Taxi drivers and chauffeurs & 1870/1937 & $67 / 57$ & $1.2(0.8-1.8)$ & $1.2(0.8-1.7)$ & $24 / 29$ & $0.8(0.4-1.4)$ & $0.8(0.5-1.4)$ & $43 / 28$ & $1.8(1.0-3.2)^{b}$ & $1.6(0.9-2.6)$ \\
\hline 9175129: Solid waste collection truck driver (motor trans.) & 1935/1985 & $2 / 9$ & $0.2(0.0-0.9)^{b}$ & $0.6(0.3-1.5)$ & $2 / 7$ & $0.2(0.0-1.2)$ & $0.7(0.3-1.6)$ & $0 / 2$ & & \\
\hline 9179118: Dispatcher, motor vehicles (motor trans.) & 1925/1991 & $12 / 3$ & $8.4(1.2-57.6)^{b}$ & $1.6(0.6-3.8)$ & $9 / 2$ & $6.8(0.9-53.9)$ & $1.4(0.6-3.5)$ & $3 / 1$ & $18.6(0.22 .5)$ & \\
\hline $\begin{array}{l}\text { 9918: Occupations in labouring and other elemental } \\
\text { work, n.e.c. }\end{array}$ & 1920/1982 & $17 / 12$ & $2.7(0.9-7.9)$ & $1.6(0.8-3.3)$ & $12 / 7$ & $4.1(1.0-16.6)^{b}$ & $1.6(0.7-3.6)$ & $5 / 5$ & $1.3(0.2-6.7)$ & $1.1(0.5-2.5)$ \\
\hline 9918110: Labourer, municipal (gov. Serv.) & 1920/1983 & $17 / 11$ & $3.4(1.0-10.8)^{\mathrm{b}}$ & $1.7(0.8-3.5)$ & $12 / 6$ & $6.5(1.2-33.5)^{b}$ & $1.6(0.7-3.8)$ & $5 / 5$ & $1.3(0.2-6.7)$ & $1.1(0.5-2.5)$ \\
\hline
\end{tabular}

accupations selected had at least one significantly elevated or reduced association with prostate cancer among duration of employment categories (ever, $<10$ years or $\geq 10$ years). Odds ratios adjusted for age, first-degree family history of prostate cancer, ancestry, screening for prostate cancer, annual household income, highest level of education attained, level of physical activity, alcohol intake and body mass index

${ }^{\text {bThe }} 95 \%$ confidence interval excludes the null value. n.e.c.: not elsewhere classified 
Table 3 Associations between selected occupations and risk of high grade prostate cancer ${ }^{\mathrm{a}}$

\begin{tabular}{|c|c|c|c|c|c|c|c|c|c|c|}
\hline \multirow[t]{2}{*}{ CCDO code and description } & \multirow{2}{*}{$\begin{array}{l}\text { Never } \\
\mathrm{Ca} / \mathrm{Co}\end{array}$} & \multicolumn{3}{|c|}{ Ever employed } & \multicolumn{3}{|c|}{$<10$ years employed } & \multicolumn{3}{|c|}{$\geq 10$ years employed } \\
\hline & & $\mathrm{Ca} / \mathrm{Co}$ & OR $(95 \% \mathrm{Cl})$ & OR SB (95\% Cl) & $\mathrm{Ca} / \mathrm{Co}$ & OR $(95 \% \mathrm{Cl})$ & OR SB (95\% Cl) & $\mathrm{Ca} / \mathrm{Co}$ & OR $(95 \% \mathrm{Cl})$ & OR SB $(95 \% \mathrm{Cl})$ \\
\hline 1135122: Credit manager (prof. \& tech., n.e.c.) & $520 / 1990$ & $4 / 4$ & $4.0(1.0-17.0)$ & $1.6(0.7-3.7)$ & $2 / 1$ & $12.6(1.1 .4)^{\mathrm{b}}$ & & $2 / 3$ & $2.2(0.3-13.8)$ & $1.3(0.6-3.1)$ \\
\hline 1137: Sales and advertising management occupations & $505 / 1931$ & $19 / 63$ & $1.3(0.7-2.2)$ & $1.2(0.8-2.0)$ & $12 / 22$ & $2.1(1.0-4.5)^{b}$ & $1.7(0.9-3.1)$ & $7 / 41$ & $0.7(0.3-1.7)$ & $0.9(0.5-1.7)$ \\
\hline 1137118: Manager, sales (prof. \& tech., n.e.c.) & $508 / 1945$ & $16 / 49$ & $1.4(0.8-2.6)$ & $1.3(0.8-2.2)$ & 10/17 & $2.8(1.2-6.5)^{b}$ & $1.9(1.0-3.6)^{b}$ & $6 / 32$ & $0.7(0.3-1.8)$ & $0.9(0.5-1.8)$ \\
\hline 2163: Draughtsmen & $512 / 1949$ & $12 / 45$ & $1.2(0.6-2.4)$ & $1.2(0.7-2.0)$ & $8 / 15$ & $2.6(1.0-6.6)^{b}$ & $1.8(0.9-3.4)$ & $4 / 30$ & $0.6(0.2-1.7)$ & $0.8(0.4-1.7)$ \\
\hline 23: Occupations in social sciences and related fields & $494 / 1914$ & $30 / 80$ & $1.8(1.1-3.0)^{b}$ & $1.7(1.1-2.6)^{b}$ & $17 / 30$ & $2.4(1.3-4.6)^{b}$ & $1.9(1.1-3.3)^{b}$ & $13 / 50$ & $1.4(0.7-2.7)$ & $1.3(0.8-2.3)$ \\
\hline 2349: Occupations in law and jurisprudence, n.e.c. & $520 / 1990$ & $4 / 4$ & $4.3(0.9-19.9)$ & $1.6(0.7-3.7)$ & $4 / 1$ & $12.2(1.3 .2)^{b}$ & $1.7(0.7-4.1)$ & $0 / 3$ & & \\
\hline 2711199: Other university teachers & $520 / 1992$ & $4 / 2$ & $7.0(1.2-39.6)^{b}$ & $1.7(0.7-4.0)$ & $3 / 2$ & $4.8(0.8-29.4)$ & $1.6(0.7-3.7)$ & $1 / 0$ & & \\
\hline 279: Other teaching and related occupations & $501 / 1933$ & $23 / 61$ & $1.7(1.0-2.8)$ & $1.5(1.0-2.4)$ & $10 / 38$ & $1.2(0.6-2.6)$ & $1.2(0.7-2.2)$ & $13 / 23$ & $2.3(1.1-4.8)^{b}$ & $1.8(1.0-3.2)$ \\
\hline 4131134: Accounting clerk (clerical) & $514 / 1976$ & 10/18 & $2.8(1.2-6.8)^{b}$ & $1.8(1.0-3.5)$ & $7 / 14$ & $2.4(0.9-6.5)$ & $1.6(0.8-3.3)$ & $3 / 4$ & $4.8(0.8-28.6)$ & $1.6(0.7-3.7)$ \\
\hline 4131142: Bookkeeping clerk (clerical) & $513 / 1973$ & $11 / 21$ & $2.8(1.2-6.3)^{b}$ & $1.9(1.0-3.6)^{b}$ & 9/15 & $3.3(1.3-8.5)^{b}$ & $2.0(1.0-3.9)^{b}$ & $2 / 6$ & $1.6(0.3-9.1)$ & $1.2(0.5-2.9)$ \\
\hline 4133110: Teller (bank. \& finance) & $512 / 1969$ & $12 / 25$ & $2.1(1.0-4.4)$ & $1.6(0.9-3.0)$ & $12 / 24$ & $2.1(1.0-4.4)^{b}$ & $1.7(0.9-3.0)$ & $0 / 1$ & & \\
\hline 5133114: Pharmaceutical representative (whole. Trade) & $520 / 1986$ & $4 / 8$ & $1.9(0.6-6.5)$ & $1.3(0.6-2.9)$ & $4 / 4$ & $4.6(1.1-19.3)^{b}$ & $1.8(0.8-4.0)$ & $0 / 4$ & & \\
\hline $\begin{array}{l}\text { 5135178: Salesperson, wearing apparel (ret. Trade; } \\
\text { whole. Trade) }\end{array}$ & $514 / 1980$ & $10 / 14$ & $3.2(1.3-7.6)^{b}$ & $2.0(1.0-3.8)^{b}$ & $7 / 10$ & $3.0(1.1-8.3)^{b}$ & $1.8(0.9-3.6)$ & $3 / 4$ & $3.8(0.7-19.4)$ & $1.6(0.7-3.6)$ \\
\hline 5145: Service station attendants & $511 / 1982$ & $13 / 12$ & $4.3(1.8-10.4)^{b}$ & $2.4(1.2-4.5)^{b}$ & $11 / 11$ & $4.0(1.6-10.2)^{b}$ & $2.2(1.1-4.3)^{b}$ & $2 / 1$ & $7.0(0.6-78.7)$ & \\
\hline $\begin{array}{l}\text { 5145110: Service-station attendant (motor vehicle; } \\
\text { ret. Trade) }\end{array}$ & $511 / 1982$ & $13 / 12$ & $4.4(1.8-10.5)^{b}$ & $2.4(1.2-4.5)^{b}$ & $11 / 11$ & $4.0(1.6-10.3)^{\mathrm{b}}$ & $2.2(1.1-4.3)^{\mathrm{b}}$ & $2 / 1$ & $7.5(0.7-84.2)$ & \\
\hline 5172: Real estate salesmen & $508 / 1960$ & $16 / 34$ & $1.8(0.9-3.3)$ & $1.5(0.9-2.6)$ & $10 / 16$ & $2.4(1.0-5.5)^{b}$ & $1.8(0.9-3.3)$ & $6 / 18$ & $1.2(0.5-3.3)$ & $1.2(0.6-2.4)$ \\
\hline 5172118: Salesperson, real estate (insur. \& real estate) & $508 / 1965$ & $16 / 29$ & $2.0(1.1-3.9)^{\mathrm{b}}$ & $1.7(1.0-2.9)$ & $10 / 14$ & $2.9(1.2-6.8)^{b}$ & $1.9(1.0-3.7)$ & $6 / 15$ & $1.4(0.5-3.7)$ & $1.2(0.6-2.5)$ \\
\hline 6112: Police officers and detectives, government & $513 / 1964$ & $11 / 30$ & $1.4(0.7-2.9)$ & $1.3(0.7-2.3)$ & $5 / 5$ & $4.0(1.0-15.1)^{b}$ & $1.8(0.8-3.9)$ & $6 / 25$ & $0.9(0.4-2.3)$ & $1.0(0.5-2.0)$ \\
\hline 75: Forestry and logging occupations & $500 / 1964$ & $24 / 30$ & $2.5(1.4-4.5)^{b}$ & $2.0(1.2-3.3)^{b}$ & $15 / 23$ & $2.0(1.0-4.0)$ & $1.6(0.9-2.9)$ & $9 / 7$ & $4.4(1.5-12.7)^{b}$ & $2.1(1.0-4.3)^{\mathrm{b}}$ \\
\hline 751: Forestry and logging occupations & $500 / 1964$ & $24 / 30$ & $2.5(1.4-4.5)^{\mathrm{b}}$ & $2.0(1.2-3.3)^{b}$ & $15 / 23$ & $2.0(1.0-4.0)$ & $1.6(0.9-2.9)$ & $9 / 7$ & $4.4(1.5-12.7)^{b}$ & $2.1(1.0-4.3)^{\mathrm{b}}$ \\
\hline 7513: Timber cutting and related occupations & $504 / 1969$ & $20 / 25$ & $2.5(1.3-4.7)^{b}$ & $1.9(1.1-3.3)^{b}$ & $12 / 20$ & $1.8(0.9-4.0)$ & $1.5(0.8-2.8)$ & $8 / 5$ & $5.2(1.5-17.6)^{b}$ & $2.1(1.0-4.4)$ \\
\hline 7513122: Logger, all-round (forest. \& log.) & $504 / 1970$ & $20 / 24$ & $2.6(1.4-5.0)^{b}$ & $2.0(1.2-3.4)^{\mathrm{b}}$ & $12 / 19$ & $2.0(0.9-4.2)$ & $1.6(0.9-2.9)$ & $8 / 5$ & $5.2(1.5-17.6)^{b}$ & $2.1(1.0-4.4)$ \\
\hline 8131: Metal smelting, converting and refining furnacemen & $522 / 1991$ & $2 / 3$ & $2.3(0.3-16.8)$ & $1.3(0.5-3.0)$ & $1 / 2$ & $1.1(0.1-12.7)$ & & $1 / 1$ & $53.7(13.2 .3)^{b}$ & \\
\hline 821/822: Food, beverage and related processing occupations & $503 / 1882$ & $21 / 112$ & $0.5(0.3-0.9)^{b}$ & $0.6(0.4-1.0)^{b}$ & $12 / 74$ & $0.5(0.2-0.9)^{b}$ & $0.6(0.4-1.1)$ & $9 / 38$ & $0.6(0.3-1.4)$ & $0.8(0.4-1.5)$ \\
\hline 825: Pulp and papermaking and related occupations & $520 / 1991$ & $4 / 3$ & $6.3(1.1-35.6)^{b}$ & $1.7(0.7-3.9)$ & $3 / 2$ & $4.2(0.7-26.3)$ & $1.5(0.6-3.6)$ & $1 / 1$ & $56.4(0.277 .5)$ & \\
\hline 826/827: Textile processing occupations & 499/1948 & $25 / 46$ & $1.8(1.0-3.2)^{\mathrm{b}}$ & $1.6(1.0-2.6)$ & $15 / 29$ & $1.5(0.8-2.9)$ & $1.4(0.8-2.4)$ & $10 / 17$ & $2.8(1.1-6.8)^{\mathrm{b}}$ & $1.8(0.9-3.6)$ \\
\hline $\begin{array}{l}\text { 8278: Occupations in labouring and other elemental work, } \\
\text { textile processing }\end{array}$ & $519 / 1991$ & $5 / 3$ & $7.8(1.5-41.2)^{\mathrm{b}}$ & $1.8(0.8-4.2)$ & $4 / 3$ & $6.2(1.1-35.2)^{b}$ & $1.7(0.7-4.0)$ & $1 / 0$ & & \\
\hline 8551: Patternmaking, marking and cutting occupations & $507 / 1959$ & $17 / 35$ & $1.6(0.9-3.0)$ & $1.4(0.8-2.4)$ & $6 / 24$ & $0.9(0.3-2.2)$ & $1.0(0.5-1.9)$ & $11 / 11$ & $3.3(1.3-8.6)^{b}$ & $2.0(1.0-3.9)^{\mathrm{b}}$ \\
\hline 8595: Painting and decorating occupations, except & $514 / 1975$ & $10 / 19$ & $1.7(0.8-3.9)$ & $1.4(0.8-2.7)$ & $8 / 9$ & $2.9(1.0-8.1)^{b}$ & $1.8(0.9-3.6)$ & $2 / 10$ & $0.6(0.1-3.2)$ & $1.0(0.4-2.2)$ \\
\hline
\end{tabular}


Table 3 Associations between selected occupations and risk of high grade prostate cancer ${ }^{\mathrm{a}}$ (Continued)

873: Electrical power, lighting and wire communications equipment erecting, installing and repairing occupations

8799: Other construction trades occupations, n.e.c.

$\begin{array}{llllllllll}498 / 1924 & 26 / 70 & 1.4(0.9-2.3) & 1.4(0.9-2.1) & 12 / 28 & 2.2(1.0-4.7)^{\mathrm{b}} & 1.7(0.9-3.1) & 14 / 42 & 1.1(0.6-2.1) & 1.1(0.6-1.9) \\ 505 / 1948 & 19 / 46 & 2.0(1.0-3.6)^{\mathrm{b}} & 1.7(1.0-2.8) & 12 / 26 & 1.9(0.9-4.1) & 1.6(0.8-2.9) & 7 / 20 & 2.0(0.7-5.6) & 1.5(0.7-3.0) \\ 507 / 1954 & 17 / 40 & 1.6(0.9-3.0) & 1.5(0.9-2.5) & 9 / 14 & 2.9(1.1-7.3)^{\mathrm{b}} & 1.9(0.9-3.6) & 8 / 26 & 1.1(0.5-2.5) & 1.1(0.6-2.1) \\ 507 / 1954 & 17 / 40 & 1.6(0.9-3.0) & 1.5(0.9-2.5) & 9 / 14 & 2.9(1.1-7.3)^{\mathrm{b}} & 1.9(0.9-3.6) & 8 / 26 & 1.1(0.5-2.5) & 1.1(0.6-2.1) \\ 519 / 1991 & 5 / 3 & 12.7(1.6 .5)^{\mathrm{b}} & 1.7(0.7-4.2) & 5 / 2 & 13.1(1.5 .1)^{\mathrm{b}} & 1.7(0.7-4.2) & 0 / 1 & & \\ 519 / 1989 & 5 / 5 & 5.1(1.2-21.5)^{\mathrm{b}} & 1.8(0.8-4.0) & 2 / 3 & 3.6(0.5-28.4) & 1.4(0.6-3.4) & 3 / 2 & 8.5(0.9-81.2) & 1.6(0.6-3.8) \\ 515 / 1985 & 9 / 9 & 3.5(1.4-9.2)^{\mathrm{b}} & 2.0(1.0-4.0)^{\mathrm{b}} & 7 / 6 & 3.8(1.2-11.6)^{\mathrm{b}} & 1.9(0.9-4.0) & 2 / 3 & 2.8(0.5-17.4) & 1.4(0.6-3.3)\end{array}$

9171110: Bus driver (motor trans.)

9179118: Dispatcher, motor vehicles (motor trans)

9310: Foremen/women, material handling and related occupations, n.e.c.

955: Electronic and related communications equipment

${ }^{a}$ High grade defined by Gleason score $\geq 8$ or $4+3$. Occupations selected had at least one significantly elevated or reduced association with prostate cancer among duration of employment categories (ever, $<10$ years or $\geq 10$ years). Odds ratios estimated using multinomial models adjusted for age, first-degree family history of prostate cancer, ancestry, screening for prostate cancer, annual household income, highest level of education attained, level of physical activity, alcohol intake (drink-years) and body mass index

${ }^{\mathrm{b}}$ The $95 \%$ confidence interval excludes the null value. n.e.c.: not elsewhere classified 
Table 4 Associations between selected industries and risk of prostate cancer (all cancers) ${ }^{a}$

\begin{tabular}{|c|c|c|c|c|c|c|c|c|c|c|}
\hline \multirow[t]{2}{*}{ SIC code and description } & \multirow{2}{*}{$\begin{array}{l}\text { Never } \\
\mathrm{Ca} / \mathrm{Co}\end{array}$} & \multicolumn{3}{|c|}{ Ever employed } & \multicolumn{3}{|c|}{$<10$ years employed } & \multicolumn{3}{|c|}{$\geq 10$ years employed } \\
\hline & & $\mathrm{Ca} / \mathrm{Co}$ & OR $(95 \% \mathrm{Cl})$ & OR SB $(95 \% \mathrm{Cl})$ & $\mathrm{Ca} / \mathrm{Co}$ & OR $(95 \% \mathrm{Cl})$ & OR SB $(95 \% \mathrm{Cl})$ & $\mathrm{Ca} / \mathrm{Co}$ & OR $(95 \% \mathrm{Cl})$ & OR SB $(95 \% \mathrm{Cl})$ \\
\hline 01: Agricultural industries & $1864 / 1886$ & $73 / 108$ & $0.6(0.4-0.9)^{b}$ & $0.6(0.5-0.9)^{b}$ & $43 / 66$ & $0.6(0.4-0.9)^{b}$ & $0.7(0.4-1.0)^{b}$ & $30 / 42$ & $0.6(0.4-1.1)$ & $0.7(0.4-1.1)$ \\
\hline 0119: Livestock combination farms & 1932/1980 & $5 / 14$ & $0.3(0.1-0.9)^{b}$ & $0.6(0.3-1.3)$ & $2 / 7$ & $0.2(0.0-1.0)$ & $0.7(0.3-1.6)$ & $3 / 7$ & $0.4(0.1-2.4)$ & $0.8(0.3-1.9)$ \\
\hline 04: Logging industry & $1881 / 1962$ & $56 / 32$ & $1.7(1.0-2.8)^{\mathrm{b}}$ & $1.5(1.0-2.4)$ & $43 / 23$ & $1.7(0.9-2.9)$ & $1.5(0.9-2.4)$ & $13 / 9$ & $1.8(0.7-4.7)$ & $1.4(0.7-2.7)$ \\
\hline 041: Logging industry & 1881/1962 & $56 / 32$ & $1.7(1.0-2.8)^{\mathrm{b}}$ & $1.5(1.0-2.4)$ & $43 / 23$ & $1.7(0.9-2.9)$ & $1.5(0.9-2.4)$ & $13 / 9$ & $1.8(0.7-4.7)$ & $1.4(0.7-2.7)$ \\
\hline 103: Fruit and vegetable industries & 1936/1985 & $1 / 9$ & $0.1(0.0-0.5)^{b}$ & $0.6(0.3-1.5)$ & $1 / 7$ & $0.1(0.0-0.8)^{b}$ & $0.7(0.3-1.6)$ & $0 / 2$ & & \\
\hline 1031: Canned and preserved fruit and vegetable industry & 1936/1985 & $1 / 9$ & $0.1(0.0-0.5)^{b}$ & $0.6(0.3-1.5)$ & $1 / 7$ & $0.1(0.0-0.8)^{b}$ & $0.7(0.3-1.6)$ & $0 / 2$ & & \\
\hline 2619: Other household furniture industries & 1922/1989 & $15 / 5$ & $3.9(1.1-13.4)^{b}$ & $1.7(0.8-3.7)$ & $11 / 5$ & $3.1(0.8-11.0)$ & $1.5(0.7-3.4)$ & $4 / 0$ & & \\
\hline 27: Paper and allied products industries & 1874/1943 & $63 / 51$ & $1.3(0.9-2.0)$ & $1.3(0.9-1.9)$ & $40 / 37$ & $1.0(0.6-1.7)$ & $1.0(0.6-1.6)$ & $23 / 14$ & $2.3(1.1-5.0)^{\mathrm{b}}$ & $1.7(0.9-3.1)$ \\
\hline 2711: Pulp industry & 1911/1972 & $26 / 22$ & $1.3(0.7-2.4)$ & $1.2(0.7-2.0)$ & $16 / 18$ & $0.8(0.4-1.7)$ & $0.9(0.5-1.6)$ & $10 / 4$ & $5.6(1.1-27.5)^{b}$ & $1.6(0.7-3.8)$ \\
\hline $\begin{array}{l}\text { 3039: Other ornamental and architectural metal } \\
\text { products industries }\end{array}$ & 1927/1991 & $10 / 3$ & $5.9(1.0-33.6)^{b}$ & $1.6(0.7-3.7)$ & $7 / 2$ & $13.2(1.3 .8)^{b}$ & $1.5(0.6-3.7)$ & $3 / 1$ & $1.6(0.2-16.1)$ & \\
\hline 3199: Other machinery and equipment industries n.e.c. & 1920/1966 & $17 / 28$ & $0.6(0.3-1.1)$ & $0.7(0.4-1.2)$ & $11 / 22$ & $0.4(0.2-1.0)^{b}$ & $0.6(0.3-1.1)$ & $6 / 6$ & $1.0(0.3-3.6)$ & $1.0(0.5-2.2)$ \\
\hline 32: Transportation equipment industries & $1809 / 1831$ & $128 / 163$ & $0.8(0.6-1.0)$ & $0.8(0.6-1.0)$ & $80 / 90$ & $0.9(0.6-1.2)$ & $0.9(0.6-1.2)$ & $48 / 73$ & $0.7(0.4-1.0)^{b}$ & $0.7(0.5-1.0)$ \\
\hline 321: Aircraft and aircraft parts industry & $1881 / 1897$ & $56 / 97$ & $0.6(0.4-0.9)^{b}$ & $0.6(0.5-0.9)^{b}$ & $32 / 48$ & $0.7(0.4-1.2)$ & $0.8(0.5-1.2)$ & $24 / 49$ & $0.5(0.3-0.8)^{b}$ & $0.6(0.4-0.9)^{b}$ \\
\hline 3211: Aircraft and aircraft parts industry & $1881 / 1897$ & $56 / 97$ & $0.6(0.4-0.9)^{b}$ & $0.6(0.5-0.9)^{b}$ & $32 / 48$ & $0.7(0.4-1.2)$ & $0.8(0.5-1.2)$ & $24 / 49$ & $0.5(0.3-0.8)^{b}$ & $0.6(0.4-0.9)^{b}$ \\
\hline 354: Concrete products industries & 1925/1987 & $12 / 7$ & $4.4(1.0-18.5)^{b}$ & $1.6(0.7-3.7)$ & $7 / 6$ & $2.3(0.5-11.2)$ & $1.3(0.6-3.0)$ & $5 / 1$ & $28.1(0.89 .3)$ & $1.3(0.5-3.3)$ \\
\hline 356: Glass and glass products industries & 1925/1976 & $12 / 18$ & $0.6(0.3-1.4)$ & $0.8(0.4-1.4)$ & $9 / 9$ & $1.2(0.4-3.4)$ & $1.1(0.5-2.2)$ & $3 / 9$ & $0.2(0.1-1.0)^{\mathrm{b}}$ & $0.6(0.3-1.4)$ \\
\hline 3561: Primary glass and glass containers industry & 1929/1977 & $8 / 17$ & $0.5(0.2-1.2)$ & $0.7(0.3-1.3)$ & $6 / 9$ & $0.9(0.3-2.8)$ & $1.0(0.4-2.0)$ & $2 / 8$ & $0.2(0.0-0.9)^{b}$ & $0.6(0.3-1.5)$ \\
\hline $\begin{array}{l}\text { 424: Plumbing, heating and air conditioning, } \\
\text { mechanical work }\end{array}$ & 1887/1957 & $50 / 37$ & $1.7(1.0-2.8)^{b}$ & $1.5(1.0-2.4)$ & 23/16 & $1.7(0.8-3.6)$ & $1.4(0.8-2.6)$ & $27 / 21$ & $1.7(0.8-3.3)$ & $1.4(0.8-2.5)$ \\
\hline 427: Interior and finishing work & 1891/1958 & $46 / 36$ & $1.7(1.0-2.9)^{\mathrm{b}}$ & $1.5(1.0-2.4)$ & $17 / 13$ & $1.8(0.8-4.4)$ & $1.4(0.7-2.7)$ & $29 / 23$ & $1.6(0.9-3.2)$ & $1.4(0.8-2.4)$ \\
\hline 451: Air transport industries & 1923/1957 & $14 / 37$ & $0.4(0.2-0.9)^{b}$ & $0.6(0.3-1.0)$ & $4 / 10$ & $0.5(0.1-1.9)$ & $0.8(0.4-1.8)$ & $10 / 27$ & $0.4(0.2-0.9)^{b}$ & $0.6(0.3-1.1)$ \\
\hline 4511: Scheduled air transport industry & 1923/1959 & $14 / 35$ & $0.5(0.2-0.9)^{b}$ & $0.6(0.3-1.0)$ & $4 / 9$ & $0.6(0.1-2.4)$ & $0.9(0.4-1.9)$ & $10 / 26$ & $0.4(0.2-0.9)^{b}$ & $0.6(0.3-1.1)$ \\
\hline 454: Water transport industries & 1925/1964 & $12 / 30$ & $0.4(0.2-0.8)^{b}$ & $0.6(0.3-1.0)$ & $6 / 21$ & $0.3(0.1-0.8)^{b}$ & $0.6(0.3-1.1)$ & $6 / 9$ & $0.6(0.2-2.0)$ & $0.8(0.4-1.8)$ \\
\hline 4541: Freight and passenger water transport industry & 1925/1966 & $12 / 28$ & $0.4(0.2-0.9)^{b}$ & $0.6(0.3-1.0)$ & $6 / 19$ & $0.3(0.1-0.8)^{b}$ & $0.6(0.3-1.1)$ & $6 / 9$ & $0.6(0.2-2.0)$ & $0.8(0.4-1.8)$ \\
\hline 4571: Urban transit systems industry & 1888/1949 & $49 / 45$ & $1.1(0.7-1.8)$ & $1.1(0.7-1.6)$ & $21 / 11$ & $2.4(1.0-5.8)^{b}$ & $1.7(0.9-3.2)$ & $28 / 34$ & $0.8(0.5-1.4)$ & $0.8(0.5-1.4)$ \\
\hline 5512: Trucks and buses, wholesale & 1932/1986 & $5 / 8$ & $0.5(0.1-1.8)$ & $0.8(0.4-1.7)$ & $1 / 7$ & $0.1(0.0-1.0)^{b}$ & $0.7(0.3-1.8)$ & $4 / 1$ & $2.5(0.3-23.3)$ & $1.2(0.5-2.9)$ \\
\hline 561: Metal and metal products, wholesale & 1933/1981 & $4 / 13$ & $0.3(0.1-1.1)$ & $0.6(0.3-1.4)$ & $1 / 9$ & $0.1(0.0-0.8)^{b}$ & $0.7(0.3-1.7)$ & $3 / 4$ & $0.9(0.2-4.5)$ & $1.0(0.4-2.3)$ \\
\hline 599: Other products n.e.c., wholesale & 1919/1985 & $18 / 9$ & $2.5(1.0-6.0)$ & $1.6(0.8-3.2)$ & $11 / 4$ & $4.9(1.1-21.6)^{b}$ & $1.6(0.7-3.7)$ & $7 / 5$ & $1.4(0.4-4.6)$ & $1.1(0.5-2.5)$ \\
\hline 5999: Other products n.e.c., wholesale & 1922/1988 & $15 / 6$ & $3.4(1.1-10.4)^{b}$ & $1.7(0.8-3.6)$ & $8 / 3$ & $6.5(0.9-46.1)$ & $1.5(0.6-3.6)$ & $7 / 3$ & $2.2(0.5-8.9)$ & $1.3(0.6-2.9)$ \\
\hline 611: Shoe stores & 1931/1980 & $6 / 14$ & $0.3(0.1-1.0)^{b}$ & $0.6(0.3-1.3)$ & $4 / 8$ & $0.3(0.1-1.2)$ & $0.7(0.3-1.5)$ & $2 / 6$ & $0.4(0.1-2.1)$ & $0.8(0.3-1.9)$ \\
\hline 6111: Shoe stores & 1931/1980 & $6 / 14$ & $0.3(0.1-1.0)^{b}$ & $0.6(0.3-1.3)$ & $4 / 8$ & $0.3(0.1-1.2)$ & $0.7(0.3-1.5)$ & $2 / 6$ & $0.4(0.1-2.1)$ & $0.8(0.3-1.9)$ \\
\hline
\end{tabular}


Table 4 Associations between selected industries and risk of prostate cancer (all cancers) ${ }^{a}$ (Continued)

\begin{tabular}{|c|c|c|c|c|c|c|c|c|c|c|}
\hline 70: Deposit accepting intermediary industries & 1834/1912 & 103/82 & $1.3(0.9-1.9)$ & $1.3(0.9-1.8)$ & $54 / 41$ & $1.7(1.0-2.7)^{b}$ & $1.5(1.0-2.4)$ & 49/41 & $1.1(0.7-1.7)$ & $1.1(0.7-1.6)$ \\
\hline $\begin{array}{l}\text { 702: Chartered banks and other banking-type } \\
\text { intermediaries }\end{array}$ & 1840/1919 & $97 / 75$ & $1.4(1.0-2.0)$ & $1.4(1.0-1.9)$ & $53 / 39$ & $1.8(1.1-2.9)^{b}$ & $1.6(1.0-2.4)^{b}$ & $44 / 36$ & $1.1(0.7-1.8)$ & $1.1(0.7-1.7)$ \\
\hline 7021: Chartered banks & $1843 / 1921$ & $94 / 73$ & $1.4(1.0-2.0)$ & $1.3(1.0-1.9)$ & $53 / 38$ & $1.8(1.1-2.9)^{b}$ & $1.6(1.0-2.5)^{b}$ & $41 / 35$ & $1.1(0.7-1.8)$ & $1.1(0.7-1.6)$ \\
\hline 72: Investment intermediary industries & 1916/1977 & $21 / 17$ & $1.9(0.9-4.1)$ & $1.5(0.8-2.7)$ & $14 / 7$ & $3.7(1.1-12.2)^{b}$ & $1.7(0.8-3.6)$ & $7 / 10$ & $1.0(0.3-2.9)$ & $1.0(0.5-2.1)$ \\
\hline 8153: Taxation administration, federal & 1922/1986 & $15 / 8$ & $3.2(1.0-9.5)^{b}$ & $1.7(0.8-3.5)$ & $7 / 4$ & $4.9(0.7-33.8)$ & $1.4(0.6-3.4)$ & $8 / 4$ & $2.4(0.6-9.4)$ & $1.4(0.6-3.1)$ \\
\hline 82: Provincial and territorial government service industries & 1781/1892 & $156 / 102$ & $1.5(1.1-2.0)^{b}$ & $1.5(1.1-2.0)^{b}$ & $72 / 54$ & $1.3(0.9-2.0)$ & $1.3(0.9-1.8)$ & $84 / 48$ & $1.8(1.2-2.7)^{b}$ & $1.6(1.1-2.4)^{b}$ \\
\hline 822: Protective services (provincial) & 1893/1971 & $44 / 23$ & $1.8(1.0-3.2)^{\mathrm{b}}$ & $1.6(1.0-2.5)$ & 23/14 & $1.6(0.7-3.4)$ & $1.4(0.7-2.5)$ & $21 / 9$ & $2.0(0.9-4.7)$ & $1.5(0.8-2.9)$ \\
\hline 8225: Regulatory services, provincial & 1920/1989 & $17 / 5$ & $6.1(1.5-24.8)^{b}$ & $1.8(0.8-4.1)$ & $8 / 3$ & $17.0(1.2 .8)^{b}$ & $1.4(0.6-3.6)$ & $9 / 2$ & $3.7(0.8-17.8)$ & $1.5(0.6-3.4)$ \\
\hline 825: General administrative services (provincial) & $1891 / 1966$ & $46 / 28$ & $1.7(1.0-2.8)$ & $1.5(0.9-2.4)$ & $21 / 16$ & $1.0(0.5-2.1)$ & $1.0(0.6-1.8)$ & $25 / 12$ & $3.0(1.3-7.0)^{b}$ & $1.9(1.0-3.6)$ \\
\hline 92: Food and beverage service industries & $1786 / 1796$ & $151 / 198$ & $0.7(0.6-0.9)^{b}$ & $0.8(0.6-1.0)^{b}$ & $91 / 115$ & $0.7(0.5-1.0)$ & $0.8(0.6-1.0)$ & $60 / 83$ & $0.8(0.5-1.1)$ & $0.8(0.6-1.1)$ \\
\hline 921: Food services & $1818 / 1832$ & $119 / 162$ & $0.7(0.6-1.0)^{b}$ & $0.8(0.6-1.0)^{b}$ & 75/99 & $0.7(0.5-1.0)$ & $0.7(0.5-1.0)$ & $44 / 63$ & $0.8(0.5-1.2)$ & $0.8(0.5-1.2)$ \\
\hline 9212: Restaurants, unlicensed (including drive-ins) & 1906/1931 & $31 / 63$ & $0.5(0.3-0.8)^{b}$ & $0.6(0.4-0.9)^{b}$ & $24 / 41$ & $0.6(0.3-1.0)$ & $0.7(0.4-1.1)$ & $7 / 22$ & $0.3(0.1-0.9)^{b}$ & $0.6(0.3-1.2)$ \\
\hline 984: Labour organizations & 1921/1974 & $16 / 20$ & $0.7(0.3-1.4)$ & $0.8(0.4-1.4)$ & $5 / 13$ & $0.3(0.1-0.9)^{b}$ & $0.6(0.3-1.2)$ & $11 / 7$ & $1.3(0.5-3.7)$ & $1.2(0.6-2.4)$ \\
\hline 9841: Labour organizations & $1921 / 1974$ & $16 / 20$ & $0.7(0.3-1.4)$ & $0.8(0.4-1.4)$ & $5 / 13$ & $0.3(0.1-0.9)^{b}$ & $0.6(0.3-1.2)$ & $11 / 7$ & $1.3(0.5-3.7)$ & $1.2(0.6-2.4)$ \\
\hline 986: Civic and fraternal organizations & 1928/1972 & $9 / 22$ & $0.4(0.2-0.8)^{b}$ & $0.6(0.3-1.1)$ & $7 / 13$ & $0.4(0.2-1.2)$ & $0.7(0.3-1.4)$ & 2/9 & $0.2(0.0-1.2)$ & $0.7(0.3-1.6)$ \\
\hline 9861: Civic and fraternal organizations & 1928/1972 & $9 / 22$ & $0.4(0.2-0.8)^{b}$ & $0.6(0.3-1.1)$ & $7 / 13$ & $0.4(0.2-1.2)$ & $0.7(0.3-1.4)$ & $2 / 9$ & $0.2(0.0-1.2)$ & $0.7(0.3-1.6)$ \\
\hline
\end{tabular}

a Industries selected had at least one significantly elevated or reduced association with prostate cancer among duration of employment categories (ever, $<10$ years or $\geq 10$ years). Odds ratios adjusted for age, first-degree family history of prostate cancer, ancestry, screening for prostate cancer, annual household income, highest level of education attained, level of physical activity, alcohol intake and body mass index ${ }^{\mathrm{b}}$ The $95 \%$ confidence interval excludes the null value. n.e.c.: not elsewhere classified 
Table 5 Associations between selected industries and risk of high grade prostate cancer ${ }^{a}$

\begin{tabular}{|c|c|c|c|c|c|c|c|c|c|c|}
\hline \multirow[t]{2}{*}{ SIC code and description } & \multirow{2}{*}{$\begin{array}{l}\text { Never } \\
\mathrm{Ca} / \mathrm{Co}\end{array}$} & \multicolumn{3}{|c|}{ Ever employed } & \multicolumn{3}{|c|}{$<10$ years employed } & \multicolumn{3}{|c|}{$\geq 10$ years employed } \\
\hline & & $\mathrm{Ca} / \mathrm{Co}$ & OR $(95 \% \mathrm{Cl})$ & OR SB $(95 \% \mathrm{Cl})$ & $\mathrm{Ca} / \mathrm{Co}$ & OR $(95 \% \mathrm{Cl})$ & OR SB (95\% Cl) & $\mathrm{Ca} / \mathrm{Co}$ & OR $(95 \% \mathrm{Cl})$ & OR SB $(95 \% \mathrm{Cl})$ \\
\hline 0111: Dairy farms & $518 / 1988$ & $6 / 6$ & $3.5(0.9-12.8)$ & $1.7(0.8-3.7)$ & $6 / 4$ & $4.5(1.1-18.9)^{b}$ & $1.8(0.8-4.0)$ & $0 / 2$ & & \\
\hline 016: Horticultural specialties & $520 / 1988$ & $4 / 6$ & $2.7(0.7-11.3)$ & $1.5(0.7-3.4)$ & $4 / 2$ & $9.8(1.1-87.4)^{b}$ & $1.6(0.7-4.0)$ & $0 / 4$ & & \\
\hline 04: Logging industry & $503 / 1962$ & $21 / 32$ & $2.1(1.1-3.9)^{b}$ & $1.8(1.1-3.0)^{\mathrm{b}}$ & $14 / 23$ & $1.8(0.9-3.7)$ & $1.5(0.9-2.8)$ & $7 / 9$ & $3.1(1.0-9.3)^{b}$ & $1.8(0.9-3.7)$ \\
\hline 041: Logging industry & $503 / 1962$ & $21 / 32$ & $2.1(1.1-3.9)^{b}$ & $1.8(1.1-3.0)^{b}$ & $14 / 23$ & $1.8(0.9-3.7)$ & $1.5(0.9-2.8)$ & $7 / 9$ & $3.1(1.0-9.3)^{b}$ & $1.8(0.9-3.7)$ \\
\hline 0412: Contract logging industry & $518 / 1986$ & $6 / 8$ & $3.7(1.1-12.5)^{b}$ & $1.8(0.8-3.9)$ & $4 / 6$ & $4.2(0.9-19.0)$ & $1.7(0.8-3.8)$ & $2 / 2$ & $2.7(0.3-21.3)$ & \\
\hline 104: Dairy products industries & $512 / 1980$ & $12 / 14$ & $2.5(1.0-6.0)^{b}$ & $1.7(0.9-3.3)$ & $7 / 11$ & $1.7(0.6-5.2)$ & $1.4(0.6-2.8)$ & $5 / 3$ & $5.3(1.0-28.0)^{b}$ & $1.7(0.7-4.0)$ \\
\hline 25: Wood industries & $504 / 1955$ & 20/39 & $1.9(1.0-3.6)^{\mathrm{b}}$ & $1.7(1.0-2.8)$ & $17 / 31$ & $2.2(1.1-4.4)^{b}$ & $1.8(1.0-3.1)^{b}$ & $3 / 8$ & $1.0(0.2-4.3)$ & $1.1(0.5-2.5)$ \\
\hline 2619: Other household furniture industries & $518 / 1989$ & $6 / 5$ & $5.5(1.4-21.9)^{\mathrm{b}}$ & $1.9(0.9-4.3)$ & $3 / 5$ & $3.0(0.6-14.7)$ & $1.5(0.6-3.4)$ & $3 / 0$ & & \\
\hline 291: Primary steel industries & $511 / 1969$ & $13 / 25$ & $2.1(1.0-4.5)^{b}$ & $1.7(0.9-3.0)$ & $6 / 17$ & $1.5(0.6-4.2)$ & $1.3(0.6-2.7)$ & $7 / 8$ & $3.2(1.1-9.8)^{b}$ & $1.8(0.9-3.8)$ \\
\hline 2919: Other primary steel industries & $518 / 1988$ & $6 / 6$ & $4.5(1.2-16.3)^{b}$ & $1.9(0.8-4.1)$ & $2 / 4$ & $3.2(0.4-23.0)$ & $1.4(0.6-3.4)$ & $4 / 2$ & $5.7(1.0-32.7)$ & $1.7(0.7-4.0)$ \\
\hline $\begin{array}{l}\text { 3039: Other ornamental and architectural } \\
\text { metal products industries }\end{array}$ & $520 / 1991$ & $4 / 3$ & $8.3(1.2-55.9)^{\mathrm{b}}$ & $1.7(0.7-4.1)$ & $4 / 2$ & $27.1(2.3 .0)^{\mathrm{b}}$ & $1.8(0.7-4.4)$ & $0 / 1$ & & \\
\hline 3331: Lighting fixture industry & $519 / 1989$ & $5 / 5$ & $5.2(1.2-21.9)^{b}$ & $1.8(0.8-4.1)$ & $3 / 4$ & $4.8(0.8-29.3)$ & $1.6(0.7-3.8)$ & $2 / 1$ & $5.8(0.5-66.3)$ & \\
\hline $\begin{array}{l}\text { 4123: Hydroelectric power plants and } \\
\text { related structures(except transmission lines) }\end{array}$ & $518 / 1987$ & $6 / 7$ & $4.9(1.3-17.7)^{\mathrm{b}}$ & $1.9(0.9-4.2)$ & $5 / 6$ & $4.3(1.1-16.4)^{b}$ & $1.8(0.8-4.0)$ & $1 / 1$ & $26.7(0.19 .5)$ & \\
\hline 4214: Excavating and grading & $516 / 1980$ & $8 / 14$ & $2.1(0.8-5.3)$ & $1.5(0.8-3.0)$ & $5 / 5$ & $5.9(1.2-29.5)^{b}$ & $1.8(0.8-4.1)$ & $3 / 9$ & $1.0(0.3-4.0)$ & $1.1(0.5-2.4)$ \\
\hline 4241: Plumbing & $512 / 1966$ & $12 / 28$ & $1.8(0.9-3.9)$ & $1.5(0.8-2.8)$ & $8 / 11$ & $3.2(1.1-8.9)^{b}$ & $1.9(0.9-3.8)$ & $4 / 17$ & $1.0(0.3-3.2)$ & $1.1(0.5-2.3)$ \\
\hline 456: Truck transport industries & $492 / 1917$ & $32 / 77$ & $1.5(1.0-2.4)$ & $1.4(0.9-2.2)$ & $7 / 35$ & $0.8(0.4-2.0)$ & $1.0(0.5-1.8)$ & $25 / 42$ & $2.0(1.1-3.5)^{b}$ & $1.7(1.1-2.8)^{b}$ \\
\hline 4571: Urban transit systems industry & $509 / 1949$ & $15 / 45$ & $1.2(0.7-2.3)$ & $1.2(0.7-2.0)$ & $8 / 11$ & $3.5(1.2-9.8)^{b}$ & $1.9(0.9-4.0)$ & $7 / 34$ & $0.7(0.3-1.7)$ & $0.9(0.5-1.7)$ \\
\hline 492: Gas distribution systems industry & $519 / 1988$ & $5 / 6$ & $3.7(1.0-13.8)^{b}$ & $1.7(0.8-3.8)$ & $1 / 2$ & $2.0(0.2-26.0)$ & & $4 / 4$ & $4.6(1.0-21.3)$ & $1.7(0.8-3.9)$ \\
\hline 4921: Gas distribution systems industry & $519 / 1988$ & $5 / 6$ & $3.7(1.0-13.8)^{\mathrm{b}}$ & $1.7(0.8-3.8)$ & $1 / 2$ & $2.0(0.2-26.0)$ & & $4 / 4$ & $4.6(1.0-21.3)$ & $1.7(0.8-3.9)$ \\
\hline 599: Other products n.e.c., wholesale & $518 / 1985$ & $6 / 9$ & $3.1(1.0-9.5)^{\mathrm{b}}$ & $1.7(0.8-3.6)$ & $2 / 4$ & $3.6(0.5-25.6)$ & $1.4(0.6-3.5)$ & $4 / 5$ & $2.8(0.7-10.9)$ & $1.5(0.7-3.4)$ \\
\hline 5999: Other products n.e.c., wholesale & $518 / 1988$ & $6 / 6$ & $5.0(1.4-18.4)^{b}$ & $1.9(0.9-4.2)$ & $2 / 3$ & $6.4(0.6-64.8)$ & $1.5(0.6-3.7)$ & $4 / 3$ & $4.3(0.9-21.0)$ & $1.7(0.7-3.8)$ \\
\hline $\begin{array}{l}\text { 6212: Household furniture stores (without } \\
\text { appliances and furnishings) }\end{array}$ & $516 / 1986$ & $8 / 8$ & $3.1(1.1-8.8)^{b}$ & $1.8(0.9-3.7)$ & $5 / 5$ & $3.2(0.8-12.3)$ & $1.6(0.7-3.6)$ & $3 / 3$ & $2.9(0.6-14.7)$ & $1.5(0.6-3.4)$ \\
\hline 633: Gasoline service stations & $506 / 1968$ & $18 / 26$ & $2.8(1.4-5.5)^{\mathrm{b}}$ & $2.1(1.2-3.6)^{b}$ & $16 / 22$ & $2.9(1.4-6.0)^{b}$ & $2.1(1.2-3.7)^{b}$ & $2 / 4$ & $2.0(0.3-11.6)$ & $1.3(0.6-3.1)$ \\
\hline 6331: Gasoline service stations & $506 / 1968$ & $18 / 26$ & $2.8(1.4-5.5)^{\mathrm{b}}$ & $2.1(1.2-3.6)^{b}$ & $16 / 22$ & $2.9(1.4-6.0)^{\mathrm{b}}$ & $2.1(1.2-3.7)^{b}$ & $2 / 4$ & $2.0(0.3-11.6)$ & $1.3(0.6-3.1)$ \\
\hline 70: Deposit accepting intermediary industries & 497/1912 & $27 / 82$ & $1.4(0.9-2.3)$ & $1.4(0.9-2.1)$ & $16 / 41$ & $1.9(1.0-3.7)^{\mathrm{b}}$ & $1.6(1.0-2.8)$ & $11 / 41$ & $1.1(0.5-2.1)$ & $1.1(0.6-1.9)$ \\
\hline $\begin{array}{l}\text { 702: Chartered banks and other banking-type } \\
\text { intermediaries }\end{array}$ & $498 / 1919$ & $26 / 75$ & $1.5(0.9-2.5)$ & $1.4(0.9-2.2)$ & $16 / 39$ & $2.0(1.1-3.9)^{\mathrm{b}}$ & $1.7(1.0-2.9)$ & $10 / 36$ & $1.1(0.5-2.3)$ & $1.1(0.6-2.0)$ \\
\hline 7021: Chartered banks & $498 / 1921$ & $26 / 73$ & $1.6(1.0-2.6)$ & $1.5(0.9-2.3)$ & $16 / 38$ & $2.1(1.1-4.0)^{\mathrm{b}}$ & $1.7(1.0-3.0)^{b}$ & $10 / 35$ & $1.2(0.6-2.4)$ & $1.1(0.6-2.1)$ \\
\hline 72: Investment intermediary industries & $517 / 1977$ & $7 / 17$ & $2.8(1.0-7.5)^{b}$ & $1.8(0.9-3.5)$ & $4 / 7$ & $4.5(1.0-19.4)^{b}$ & $1.7(0.8-3.9)$ & $3 / 10$ & $2.0(0.5-8.0)$ & $1.4(0.6-3.0)$ \\
\hline 729: Other investment intermediaries & $520 / 1989$ & $4 / 5$ & $6.0(1.3-27.7)^{b}$ & $1.8(0.8-4.2)$ & $2 / 0$ & & & $2 / 5$ & $3.0(0.5-18.3)$ & $1.4(0.6-3.4)$ \\
\hline
\end{tabular}


Table 5 Associations between selected industries and risk of high grade prostate cancer ${ }^{\text {a }}$ (Continued)

\begin{tabular}{|c|c|c|c|c|c|c|c|c|c|c|}
\hline 7299: Other investment intermediaries n.e.c. & $520 / 1990$ & $4 / 4$ & $6.1(1.3-27.9)^{b}$ & $1.8(0.8-4.2)$ & $2 / 0$ & & & $2 / 4$ & $3.0(0.5-18.3)$ & $1.4(0.6-3.4)$ \\
\hline 81: Federal government service industries & 469/1734 & $55 / 260$ & $0.8(0.6-1.1)$ & $0.8(0.6-1.1)$ & $33 / 183$ & $0.7(0.4-1.0)^{b}$ & $0.7(0.5-1.0)$ & $22 / 77$ & $1.2(0.7-1.9)$ & $1.2(0.7-1.8)$ \\
\hline $\begin{array}{l}\text { 8152: Finance and economic administration, } \\
\text { federal }\end{array}$ & $520 / 1987$ & $4 / 7$ & $7.7(1.5-38.7)^{b}$ & $1.9(0.8-4.4)$ & $1 / 5$ & $3.1(0.2-42.6)$ & $1.3(0.5-3.2)$ & $3 / 2$ & $14.4(1.6 .0)^{b}$ & $1.7(0.7-4.3)$ \\
\hline $\begin{array}{l}\text { 8164: Recreation and culture administration, } \\
\text { federal }\end{array}$ & $521 / 1993$ & $3 / 1$ & $122.3(0.554 .8)$ & & $3 / 1$ & $137.2(63.6 .9)^{b}$ & & $0 / 0$ & & \\
\hline $\begin{array}{l}\text { 82: Provincial and territorial government } \\
\text { service industries }\end{array}$ & $482 / 1892$ & $42 / 102$ & $1.7(1.2-2.6)^{b}$ & $1.6(1.1-2.4)^{b}$ & $22 / 54$ & $1.6(0.9-2.8)$ & $1.5(0.9-2.4)$ & $20 / 48$ & $1.8(1.0-3.2)^{b}$ & $1.6(1.0-2.7)^{b}$ \\
\hline 8259: Other general administrative services, provincial & $519 / 1992$ & $5 / 2$ & $8.0(1.5-42.9)^{b}$ & $1.8(0.8-4.3)$ & $3 / 1$ & $8.9(0.9-88.6)$ & & $2 / 1$ & $7.5(0.6-86.4)$ & \\
\hline 83: Local government service industries & $483 / 1856$ & $41 / 138$ & $1.2(0.8-1.7)$ & $1.1(0.8-1.6)$ & 20/44 & $1.9(1.1-3.4)^{\mathrm{b}}$ & $1.7(1.0-2.8)^{\mathrm{b}}$ & $21 / 94$ & $0.8(0.5-1.4)$ & $0.9(0.6-1.4)$ \\
\hline 8362: Social service administration, municipal & $519 / 1988$ & $5 / 6$ & $4.0(1.1-15.2)^{b}$ & $1.8(0.8-3.9)$ & $2 / 3$ & $6.6(0.7-62.7)$ & $1.5(0.6-3.7)$ & $3 / 3$ & $2.9(0.6-14.8)$ & $1.5(0.6-3.4)$ \\
\hline 855: Museums and archives & $519 / 1990$ & $5 / 4$ & $3.4(0.8-13.7)$ & $1.6(0.7-3.6)$ & $5 / 3$ & $4.9(1.1-22.4)^{b}$ & $1.8(0.8-4.0)$ & $0 / 1$ & & \\
\hline 8551: Museums and archives & $519 / 1990$ & $5 / 4$ & $3.4(0.8-13.7)$ & $1.6(0.7-3.6)$ & $5 / 3$ & $4.9(1.1-22.4)^{b}$ & $1.8(0.8-4.0)$ & $0 / 1$ & & \\
\hline 864: Non-institutional social services & $516 / 1975$ & $8 / 19$ & $1.7(0.7-4.3)$ & $1.4(0.7-2.8)$ & $6 / 11$ & $3.4(1.1-10.6)^{b}$ & $1.8(0.9-3.8)$ & $2 / 8$ & $0.6(0.1-3.0)$ & $1.0(0.4-2.2)$ \\
\hline 8642: Child welfare services & $519 / 1988$ & $5 / 6$ & $2.9(0.8-10.7)$ & $1.6(0.7-3.5)$ & $3 / 1$ & $62.2(1.83 .3)^{\mathrm{b}}$ & & $2 / 5$ & $1.0(0.2-5.6)$ & $1.1(0.5-2.6)$ \\
\hline 9212: Restaurants, unlicensed (including drive-ins) & $516 / 1931$ & $8 / 63$ & $0.4(0.2-1.0)^{b}$ & $0.6(0.3-1.2)$ & $6 / 41$ & $0.5(0.2-1.2)$ & $0.7(0.4-1.4)$ & $2 / 22$ & $0.3(0.1-1.4)$ & $0.8(0.3-1.8)$ \\
\hline $\begin{array}{l}\text { 961: Motion picture, audio and video production } \\
\text { and distribution }\end{array}$ & $518 / 1985$ & $6 / 9$ & $2.8(0.9-8.6)$ & $1.7(0.8-3.5)$ & $1 / 3$ & $1.2(0.1-11.7)$ & & $5 / 6$ & $3.9(1.0-14.8)^{b}$ & $1.8(0.8-3.9)$ \\
\hline
\end{tabular}

${ }^{a}$ High grade defined by Gleason score $\geq 8$ or $4+3$. Industries selected had at least one significantly elevated or reduced association with prostate cancer among duration of employment categories (ever, $<10$ years or $\geq 10$ years). Odds ratios estimated using multinomial models adjusted for age, first-degree family history of prostate cancer, ancestry, screening for prostate cancer, annual household income, highest level of education attained, level of physical activity, alcohol intake and body mass index

${ }^{\mathrm{b}}$ The $95 \%$ confidence interval excludes the null value. n.e.c.: not elsewhere classified 
2.1, $95 \%$ CI 1.2-3.7), Wood industries (ORsb 2.2, $95 \%$ CI 1.8, 95 \% CI 1.0-3.1) Local government (ORsb 1.7, $95 \%$ CI 1.0-2.8). Inverse associations for employment in Agriculture, Aircraft and Food and beverage industries remained statistically significant after SB adjustment.

\section{Discussion}

This study explored associations between PCa risk, including high grade tumors, and employment in a wide range of occupations and industries using data from the occupational histories of approximately 4000 subjects recruited in the general population. The major substantive findings, and how they compare with results of previous studies, are detailed in the next sections, followed by an examination of the study strengths and weaknesses.

Regarding occupations and industries associated with increased $\mathrm{PCa}$ risk, consistently positive associations for all and high grade PCa were found for Forestry and logging occupations, with the strongest ones $(\mathrm{OR} \geq 1.9)$ found for ever employment and employment $\geq 10$ years. Corresponding results for the Logging industry were slightly attenuated but still greater than 1.5. A recent case-control study in Northeastern Ontario found similar associations between PCa risk and employment in forestry and logging, with an OR of 2.70 (95\% CI 1.21-4.79) observed for employment $\geq 10$ years [28]. Other results in the literature range from a weakly positive association reported in a Swedish registry study [12] to an inverse association in a large population cohort in the Nordic countries [13]. Elevated PCa risks have also been observed for other occupations related to forestry, such as forest conservationists [29] and forest law enforcement [12] although the associations found in our study mainly concerned logging. We also observed weaker associations for the wood industry as well as the pulp and papermaking occupations and industries. Elevated incidence or mortality from $\mathrm{PCa}$ associated with pulp and papermaking occupations or industries has been reported in some studies $[12,30]$. Potential exposures associated with forestry and logging include pesticides [31], whole-body vibration [32, 33], wood dust [34], which can also be encountered in the Wood industry, and polycyclic aromatic hydrocarbons (PAHs) [35].

Police officers and detectives represent another occupational group where increased PCa risk was found throughout our study. Most of the studies recently reviewed by Wright, et al. [24] did not point towards elevated PCa incidence or mortality among police officers with the notable exception of a Dutch study which found a RR of 3.9 for the longest-held job [36]. Other studies not included in the Wright, et al. review found elevated PCa risk for these occupations [10, 37, 38], while a previous study conducted in Montreal [39] found no association between $\mathrm{PCa}$ and employment for the broader Protective services occupations, which also cover firefighting. For the latter, we did observe a statistically significantly increased risk of low grade PCa for employment $\geq 10$ years (presented in Additional file 1: Table S1) which remained significant following SB adjustment. Potential exposures of police officers and detectives include $\mathrm{PAH}$ and nonionizing radiation from radar guns [36, 40, 41], although the intensity of exposure associated with the use of radar guns is generally very low [42]. These occupations may also entail night-shift work, which has been associated with prostate cancer in the literature [43].

We also observed elevated PCa risk for Mixers and painting and decorating occupations, which are more directly involved with industrial chemicals. A previous case-control study in Montreal found no association between ever employment as a painter and PCa [44] although they did not make a distinction, like us, between construction and non-construction painters. Another study within the same population did find a positive association for painting, stripping and varnishing as a leisure activity [45]. Positive associations with $\mathrm{PCa}$ and employment in the paint and varnish industry have also been reported in another Canadian study [10]. These occupations/industries can be associated with a wide array of potential exposures, such as paints, lacquers, binding agents, pigments and solvents, as well as cadmium for which IARC considers to have limited evidence of an association with PCa [14].

There was also evidence in our study of excess $\mathrm{PCa}$ risk in several white collar occupations, including social sciences and administrative, management and clerical occupations. These typically entail few chemical exposures, but may reflect lower workplace physical activity levels, higher PCa screening practices, among other factors. We adjusted for these in our models with summary variables but it may be that residual confounding is at play. The literature is rather uninformative with respect to $\mathrm{PCa}$ risk in white collar occupations although excess risk among administrators and clerical workers has been reported [13].

We found some occupations for which elevated risks were restricted to high grade cancer, such as Bus drivers. Excess mortality from PCa for bus drivers was reported in an American study [46]. We also found elevated PCa risk for employment in the truck transport industry and, to a lesser extent, for heavy truck drivers, which have been reported in the literature, including for aggressive PCa $[17,47]$. There is some evidence that whole-body vibration might play a role in the associations between driving occupations and PCa [48], but other exposures such as diesel exhaust and PAHs [39, 49], and circadian rhythm disruption [50] may be involved. Another group was Gasoline station attendants, where the elevated risk of high grade $\mathrm{PCa}$ was mainly found for employment 
$<10$ years. Previous evidence on this is limited; a study from 1987 observed a small, non-significant increase in mortality, based on three observed deaths [51]. Finally, the increased risk of high-grade cancer observed for employment in occupations such as gasoline station attendants, textile processing and truck drivers does not appear to reflect delayed detection for lack of screening as associations remained essentially the same when restricting the study sample to men recently screened.

Regarding Farming and agriculture, a recent metaanalysis [52] found statistically significantly increased PCa risk among farmers, while another review [53] did not find conclusive evidence of increased PCa incidence associated with employment in farming. Our findings for these occupations were generally null or even negative for employment of less than 10 years, and are comparable to those from a previous case-control study in Montreal [39]. Several factors could explain these observations. First, the study population was mainly urban, focusing on Montreal residents at recruitment, which led to a limited number of subjects previously employed in agriculture. Employment in these occupations was mainly short term ( $<10$ years), prior to the mid-1970s, and a sizable proportion of subjects were European and Haitian immigrants. Second, employment was generally in small, family-run farms in field crop, vegetable and animal production. Chemicals thought to underlie associations with $\mathrm{PCa}$ in a number of previous investigations include organochlorines and organophosphates. It may well be that our study base includes fewer agricultural workers who were exposed to synthetic pesticides compared to other studies focusing on large-scale farming operations, explaining divergent findings. In addition, there is evidence that common genetic variations in xenobiotic metabolic enzymes can modulate the $\mathrm{PCa}$ risk associated with agricultural exposures [54], which was not accounted for in our analyses. Farmers may also be exposed to very high levels of ultraviolet radiation which may have a protective effect on PCa over several decades [55]. However, cumulative exposure in our study is not likely to be high due to the duration of employment of subjects ever employed as farmers was mostly less than 10 years. Finally, the generally lowered risks observed here might also reflect under-detection of $\mathrm{PCa}$ in our farmer group relative to other occupations, which was not fully captured through our consideration of screening practices.

Aside from farming, significantly reduced PCa risks were found for employment in aircraft and air transportation occupations or industries. Some studies have found some suggestive evidence of higher PCa risk among pilots $[11,46]$; in our case, the subjects employed within the Air transportation minor group were split between flight deck crew and support operations (e.g. air traffic controllers), resulting in small numbers within specific occupations. Aircraft maintenance workers may also perform work outdoors. However, reviews of the job descriptions indicate that most of them appear to have worked a significant portion of the time inside hangars, limiting exposure to UV radiation.

The potential associations between employment in other occupation groups and PCa risk have been explored by several studies, but findings for most specific occupations relied on a handful of studies, several of which were quite small. Moreover, large registry-based analyses focused on $\mathrm{PCa}$ mortality, which does not relate well to $\mathrm{PCa}$ incidence, and few assessed disease aggressiveness. Reviews on the subject concluded that the evidence remains scarce, and that methodological issues hampered interpretation. Some hints were apparent for occupations entailing metal and related exposures, oil-based fluids, petroleum, PAHs, engine emissions or PCBs, but there was little or no evidence accrued for occupation groups exposed to cadmium, lead, chromium and rubber products $[5,6]$. The complex mixtures encountered in many occupations render them non-specific with regard to exposures per se, reenforcing the need to conduct studies focusing on detailed exposure assessment protocols.

The job and industry titles in this study were derived from detailed information provided by subjects about their occupations, including specific tasks. Validity studies have generally shown high concordance between historical records of employment and self-reports [56]. Assignment of occupational codes might have also entailed errors. However, the translation of the free-text job descriptions into standardized job and industry titles was conducted by experienced chemists-hygienists blinded to the subjects' case/control status, likely minimizing inconsistencies. The coding of job titles up to the 7-digit level of the CCDO classification also provided finer employment categories than most previous studies.

Response rates in the study were relatively high for cases (79\%) and lower for controls (56\%), although these values compare reasonably well with other studies based on lengthy in-person interviews. In the event that individuals who selected themselves out of the study had characteristics related to their occupation, biased risk estimates might ensue. We compared respondents and nonrespondents for several socio-economic characteristics using census information, including education, income, proportion of recent immigrants and unemployment rates; for both cases and controls, differences were minimal. In addition, ORs for the associations between African or Asian ancestry and $\mathrm{PCa}$, relative to European, and for first-degree family history of $\mathrm{PCa}$, were comparable to those reported in the literature. These results suggest that selection bias should not be of major concern. Our study also features several important strengths, the first being the large size of the study population comprising almost 4000 subjects, making this the largest population-based 
case-control study investigating the role of occupational circumstances in PCa risk. Statistical power to detect associations in the more prevalent occupational groups was excellent, although it became limited for more specific employment categories.

Second, it relied on histologically confirmed PCa cases; for controls, screening rates were very high with over $75 \%$ reporting having been screened by PSA and/or DRE within the 2 years preceding the date of interview. In Montreal, Canada, access to the medical system is universal and free of charge. While there is no systematic PCa screening program in place, at the time of study screening was frequently integrated within the annual routine medical examination, independently of socioeconomic status. Screening behavior can be influenced by a number of factors, including lifestyle, beliefs, and medical follow-ups, including those offered in the workplace, and thus can potentially confound an occupation$\mathrm{PCa}$ association. The available information on $\mathrm{PCa}$ screening patterns in our population allowed us to adjust for screening in the models and limit the impact of disease misclassification on our results and conclusions. Moreover, analyses excluding controls not screened for $\mathrm{PCa}$ in the previous 2 years to limit the potential for latent $\mathrm{PCa}$ yielded similar findings.

Third, information on Gleason scores for cases was extracted from pathology reports and was available for nearly all subjects, allowing us to investigate potential associations stratified by PCa grade; this has generally been overlooked in studies of occupational risk factors for PCa. Fourth, the information available on recognized risk factors (e.g. ancestry, family history) and other factors (e.g. BMI, education level) associated directly or indirectly with $\mathrm{PCa}$ allowed us to account for these potential confounders in the inferences made.

Lastly, we used statistical methods to account for the large number of associations between employment by occupation or industry and PCa risk in order to identify the most robust results warranting further investigation. On average, $30 \%$ of the statistically significant ORs remained as such following SB adjustment across the different analyses performed.

Little data has been accrued to date on the role of specific occupational exposures in prostate cancer risk. Agents for which there is limited evidence for humans include arsenic and inorganic arsenic compounds, cadmium and cadmium compounds, malathion, among others [14], and a role for night work is also suspected [43]. Some of these may or may not explain some of the associations observed here, but in the present population-based analysis, job and industry titles should not be viewed as reliable proxies for specific occupational exposures. Nevertheless, emerging associations can serve as leads for future work investigating specific exposures. On one hand, the identification of positive associations between employment in a given occupation or industry and PCa risk could be due to exposure to one agent, or to a diversity of agents and mixtures, not discounting the possibility of interactions between agents. On the other hand, exposure to an agent associated with PCa might occur in a range of occupations and the association might not be picked up with an analysis based on job titles, especially if the prevalence of exposure within occupations is low. Grouping subjects according to specific exposures, rather than job titles, is thus a more powerful approach to detect associations [57]. Indeed, one of the limitations identified in reviews of occupational risk factors for $\mathrm{PCa}[5,6]$ has centered on the use of crude methods for exposure assessment in many studies. To this end, the translation of the occupational histories in the PROtEuS study into estimates of exposure to over 300 agents is currently ongoing.

\section{Conclusions}

Our findings suggest excess PCa risks among forestry workers and policemen, as well as in some predominantly white collar occupations and industries such as public service, administrative and clerical work. This represents an interesting research lead as little evidence has been accrued to date on white collar occupations and cancer risk. Increased risk of high grade $\mathrm{PCa}$ was also found for other occupations such as gas station attendants and bus drivers that were not apparent when looking at low and high PCa grades combined. Additional investigations are needed to identify specific exposures or circumstances potentially associated with $\mathrm{PCa}$ among subjects employed in these occupations.

\section{Additional files}

Additional file 1: Table S1. Associations between PCa risk (all PCa, low-grade and high-grade P(a) for ever employment and duration of employment, occupations. (XLS 702 kb)

Additional file 2: Table S3. List of occupation and industry categories featuring at least one subject ever employed and $<10$ subjects ever employed or 0 case or control. (XLS 449 kb)

Additional file 3: Table S2 Associations between PCa risk (all PCa, low-grade and high-grade P(a) for ever employment and duration of employment, industries. (XLS $600 \mathrm{~kb}$ )

\section{Abbreviations}

AHS: Agricultural Health Study; BMI: Body-mass index; CCDO: Canadian Classification and Dictionary of Occupations; Cl: Confidence interval; n.e.c.: not elsewhere classified; OR: Odds ratio; PAH: Polycyclic aromatic hydrocarbon; PCa: Prostate cancer; PSA: Prostatic specific antigen; SB: Semi-Bayes; SIC: Standardized Industrial Classification

\section{Acknowledgments}

The contribution of the entire Epidemiology and Biostatistics unit at INRSInstitut Armand-Frappier is gratefully acknowledged. In particular, we want to highlight the work of Robert Bourbonnais, Ramzan Lakhani, Louise Nadon, Dora Rodriguez and Mounia Rhazi in reviewing questionnaire data and 
coding of occupation and industry titles. We thank the urologists from the participating hospitals for their collaboration.

\section{Funding}

Funding for this study was supported by the Canadian Cancer Society (grants no. 13149, 19500, 19864, 19865), the Cancer Research Society, the Fonds de Recherche du Québec — Santé (FRQS), FRQS-RRSE and the Ministère du Dévelppement Économique, de l'Innovation et de l'Exportation du Québec. JFS was supported by the FRQS and the Institut de Recherche Robert-Sauvé en Santé et en Sécurité du Travail. MEP and JL hold career awards from the FRQS. Analyses were conducted on the supercomputer Briarée which is funded by the Canada Foundation for Innovation, NanoQuébec, RMGA and the Fonds de recherche du Québec-Nature et technologies (FRQ-NT).

\section{Availability of data and materials}

The data used for this project is not shared on public repositories for confidentiality reasons and since the analysis of the PROtEuS study is ongoing. The data can be made available for use in research collaborations by contacting the corresponding author of this manuscript.

\section{Authors' contributions}

JFS conducted the data analysis and preparation of the paper. MEP contributed to the conception, design and data acquisition of the PROtEuS study. JFS, MEP and $J \mathrm{~L}$ contributed to the interpretation of the data and critical revision of the manuscript. All authors read and approved the final manuscript.

\section{Competing interests}

The authors declare that they have no competing interests.

\section{Consent for publication}

Not applicable.

\section{Ethics approval and consent to participate}

This study was approved by the Ethics Committees of the following institutions: Institut national de la recherche scientifique, Centre de Recherche du Centre Hospitalier de I'Université de Montréal, Hôpital Maisonneuve-Rosemont, Hôpital Jean- Talon, Hôpital Fleury, and Hôpital Charles-LeMoyne. All participants provided written informed consent.

\section{Author details}

${ }^{1}$ School of Public Health, Department of environmental and occupational health, Université de Montréal, C.P. 6128 Succursale Centre-Ville, Montréal, Québec H3C 3J7, Canada. ${ }^{2}$ Centre de recherche du CHUM, 850 rue Saint-Denis, Montréal, Québec H2X 0A9, Canada. ${ }^{3}$ Epidemiology and Biostatistics UnitEpidemiology and Biostatistics Unit, INRS-Institut Armand-Frappier, Institut national de la recherche scientifique, Université du Québec, 531 Boul. des Prairies, Laval, Québec H7V 1B7, Canada. ${ }^{4}$ School of Public Health, Department of Social and Preventive Medicine, Université de Montréal, C.P. 6128 Succursale Centre-Ville, Montréal, Québec H3C 3J7, Canada.

Received: 30 July 2016 Accepted: 14 October 2016

\section{Published online: 21 October 2016}

\section{References}

1. Canadian Cancer Society's Advisory Committee on Cancer Statistics. Canadian Cancer Statistics 2015. Toronto: Canadian Cancer Society; 2015

2. Siegel RL, Miller KD, Jemal A. Cancer statistics, 2016. CA Cancer J Clin. 2016:66(1):7-30

3. Ferlay J, Soerjomataram I, Dikshit R, Eser S, Mathers C, Rebelo M, Parkin DM, Forman D, Bray F. Cancer incidence and mortality worldwide: Sources, methods and major patterns in GLOBOCAN 2012. Int J Cancer. 2015;136(5):E359-E86.

4. Patel A, Klein E. Risk factors for prostate cancer. Nat Clin Pract Urol. 2009;6(2):87-95

5. Parent M-É, Siemiatycki J. Occupation and prostate cancer. Epidemiol Rev. 2001;23(1):138-43.

6. Doolan G, Benke G, Giles $G$. An update on occupation and prostate cancer Asian Pac J Cancer Prev. 2014;15(2):501-16.

7. Cogliano VJ, Baan R, Straif K, Grosse Y, Lauby-Secretan B, El Ghissassi F, Bouvard V, Benbrahim-Tallaa L, Guha N, Freeman C, et al. Preventable exposures associated with human cancers. J Natl Cancer Inst. 2011;203(24):1827-39.
8. Alavanja MCR, Bonner MR. Occupational pesticide exposures and cance risk: a review. J Toxicol Environ Health Part B. 2012;15(4):238-63.

9. Pukkala E, Martinsen Jl, Weiderpass E, Kjaerheim K, Lynge E, Tryggvadottir L, Sparén P, Demers PA. Cancer incidence among firefighters: 45 years of follow-up in five Nordic countries. Occup Environ Med. 2014;71(6):398-404.

10. Band PR, Le ND, Fang R, Threlfall WJ, Gallagher RP. Identification of occupational cancer risks in British Columbia. Part II: A population-based case-control study of 1516 Prostatic cancer cases. J Occup Environ Med. 1999:41(4):233-47.

11. Buja A, Lange JH, Perissinotto E, Rausa G, Grigoletto F, Canova C, Mastrangelo G. Cancer incidence among male military and civil pilots and flight attendants: an analysis on published data. Toxicol Ind Health. 2005;21(9):273-82

12. Sharma-Wagner S, Chokkalingam AP, Malker HS, Stone BJ, McLaughlin JK, Hsing AW. Occupation and prostate cancer risk in Sweden. J Occup Environ Med. 2000;42(5):517-25.

13. Pukkala E, Martinsen Jl, Lynge E, Gunnarsdottir HK, Sparén P, Tryggvadottir L, Weiderpass E, Kjaerheim K. Occupation and cancer - follow-up of 15 million people in five Nordic countries. Acta Oncol. 2009:48(5):646-790.

14. Cogliano VJ, Baan R, Straif K, Grosse Y, Lauby-Secretan B, El Ghissassi F, Bouvard V, Benbrahim-Tallaa L, Guha N, Freeman C, et al. Preventable exposures associated with human cancers. J Natl Cancer Inst. 2011; 103(24):1827-39.

15. Potti A, Panwalkar AW, Langness E. Prevalence of pesticide exposure in young males $(</=50$ years) with adenocarcinoma of the prostate. $J$ Carcinog. 2003;2(1):4

16. Rybicki BA, Neslund-Dudas C, Nock NL, Schultz LR, Eklund L, Rosbolt J, Bock $\mathrm{CH}$, Monaghan KG. Prostate cancer risk from occupational exposure to polycyclic aromatic hydrocarbons interacting with the GSTP1 Ile105Val polymorphism. Cancer Detect Prev. 2006;30(5):412-22.

17. Su LJ, Fontham ETH. Prostate cancer: an occupational hazard? Future Oncol. 2014:10(6):903-6.

18. Su LJ, Sun W, Fontham ET, Bensen JT, Mohler JL. Occupational risk factors for high aggressive prostate cancer in a population-based study of African Americans and European Americans [abstract]. Cancer Prev Res. 2013:6 Suppl 11:B63.

19. Koutros S, Beane Freeman LE, Lubin JH, Heltshe SL, Andreotti G, Barry KH, DellaValle CT, Hoppin JA, Sandler DP, Lynch CF, et al. Risk of total and aggressive prostate cancer and pesticide use in the agricultural health study. Am J Epidemiol. 2013;177(1):59-74.

20. Jones RR, Barone-Adesi F, Koutros S, Lerro CC, Blair A, Lubin J, Heltshe SL, Hoppin JA, Alavanja MCR, Beane Freeman LE. Incidence of solid tumours among pesticide applicators exposed to the organophosphate insecticide diazinon in the Agricultural Health Study: an updated analysis. Occup Environ Med. 2015;72(7):496-503.

21. Blanc-Lapierre A, Spence A, Karakiewicz PI, Aprikian A, Saad F, Parent M-É. Metabolic syndrome and prostate cancer risk in a population-based casecontrol study in Montreal, Canada. BMC Public Health. 2015;18(15):913

22. Department of Employment and Immigration. Canadian classification dictionnary of occupations, vol. 1. Ottawa: Department of Employment and Immigration; 1971

23. Statistics Canada. Standard Industrial Classification, 1980. Ottawa: Statistics Canada, Standards Division; 1980

24. Wright JL, Salinas CA, Lin DW, Kolb S, Koopmeiners J, Feng Z, Stanford JL. Prostate cancer specific mortality and gleason 7 disease differences in prostate cancer outcomes between cases with gleason $4+3$ and gleason 3 + 4 tumors in a population based cohort. J Urol. 2009;182(6):2702-7.

25. Greenland S, Poole C. Empirical-bayes and semi-bayes approaches to occupational and environmental hazard surveillance. Arch Environ Health. 1994;49(1):9-16.

26. Dryson E, t Mannetje A, Walls C, McLean D, McKenzie F, Maule M, Cheng S, Cunningham $\mathrm{C}$, Kromhout $\mathrm{H}$, Boffetta $\mathrm{P}$, et al. Case-control study of high risk occupations for bladder cancer in New Zealand. Int J Cancer. 2008;122(6):1340-6.

27. Corbin M, Richiardi L, Vermeulen R, Kromhout H, Merletti F, Peters S, Simonato L, Steenland K, Pearce N, Maule M. Hierarchical regression for multiple comparisons in a case-control study of occupational risks for lung cancer. PLoS One. 2012;7(6):e38944

28. Sritharan J, Demers PA, Harris SA, Cole DC, Kreiger N, Sass-Kortsak A, Lightfoot N. Natural resource-based industries and prostate cancer risk in Northeastern Ontario: a case-control study. Occup Environ Med. 2016;73(8):506-11. 
29. Alavanja MCR, Blair A, Merkle S, Teske J, Eaton B, Reed B. Mortality among forest and soil conservationists. Arch Environ Health. 1989;44(2):94-101.

30. Band PR, Abanto Z, Bert J, Lang B, Fang R, Gallagher RP, Le ND. Prostate cancer risk and exposure to pesticides in British Columbia Farmers. Prostate. 2011;71(2):168-83.

31. Kangas J. Chemical Hazards. Chapter 68, Forestry. In: Poschen P, editor. Encyclopedia of Occupational Health and Safety. Geneva: International Labor Organization; 2011.

32. Jack RJ, Oliver M. A review of factors influencing whole-body vibration injuries in forestry mobile machine operators. Int J Forest Eng. 2008;19(1):51-65.

33. Nadalin V, Kreiger N, Parent M-E, Salmoni A, Sass-Kortsak A, Siemiatycki J, Sloan M, Purdham J. Prostate cancer and occupational whole-body vibration exposure. Ann Occup Hyg. 2012;56(8):968-74.

34. Siemiatycki J, Richardson L, Straif K, Latreille B, Lakhani R, Campbell S, Rousseau M-C, Boffetta P. Listing occupational carcinogens. Environ Health Perspect. 2004;112(15):1447-59.

35. Driscoll TR, Carey RN, Peters S, Glass DC, Benke G, Reid A, Fritschi L. The Australian work exposures study: occupational exposure to polycyclic aromatic hydrocarbons. Ann Occup Hyg. 2016;60(1):124-31.

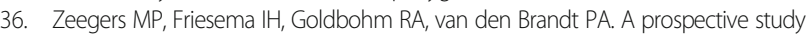
of occupation and prostate cancer risk. J Occup Environ Med. 2004:46(3):271-9.

37. van der Gulden JW, Kolk JJ, Verbeek AL. Work environment and prostate cancer risk. Prostate. 1995:27(5):250-7.

38. Finkelstein MM. Cancer incidence among Ontario police officers. Am J Ind Med. 1998;34(2):157-62.

39. Aronson KJ, Siemiatycki J, Dewar R, Gérin M. Occupational risk factors for prostate cancer: results from a case-control study in Montréal, Québec,Canada. Am J Epidemiol. 1996;143(4):363-73.

40. Wirth M, Vena JE, Smith EK, Bauer SE, Violanti J, Burch J. The epidemiology of cancer among police officers. Am J Ind Med. 2013;56(4):439-53.

41. Srogi K. Monitoring of environmental exposure to polycyclic aromatic hydrocarbons: a review. Environ Chem Lett. 2007;5(4):169-95.

42. IARC. Non-lonizing Radiation, Part 2: Radiofrequency Electromagnetic Fields, vol. 102. Lyon: International Agency for Research on Cancer; 2013.

43. Rao D, Yu H, Bai Y, Zheng $X$, Xie L. Does night-shift work increase the risk of prostate cancer? a systematic review and meta-analysis. Onco Targets Ther. 2015;8:2817-26.

44. Ramanakumar AV, Nadon L, Siemiatycki J. Exposures in painting related occupations and risk of selected cancers: Results from a case-control study in montreal. Am J Ind Med. 2008:51(6):419-27.

45. Sharpe $C R$, Siemiatycki J, Parent M-É. Activities and exposures during leisure and prostate cancer risk. Cancer Epidemiol Biomarkers Prev. 2001;10(8):855-60.

46. Krstev S, Baris D, Stewart PA, Hayes RB, Blair A, Dosemeci M. Risk for prostate cancer by occupation and industry: a 24-state death certificate study. Am J Ind Med. 1998;34(5):413-20.

47. Järvholm B, Silverman D. Lung cancer in heavy equipment operators and truck drivers with diesel exhaust exposure in the construction industry. Occup Environ Med. 2003;60(7):516-20.

48. Young E, Kreiger N, Purdham J, Sass-Kortsak A. Prostate cancer and driving occupations: could whole body vibration play a role? Int Arch Occup Environ Health. 2009;82(5):551-6.

49. Seidler A, Heiskel H, Bickeboller R, Elsner G. Association between diesel exposure at work and prostate cancer. Scand J Work Environ Health. 1998;24(6):486-94.

50. Mitler MM, Miller JC, Lipsitz JJ, Walsh JK, Wylie CD. The sleep of long-haul truck drivers. N Engl J Med. 1997;337(11):755-62.

51. Schwartz E. Proportionate mortality ratio analysis of automobile mechanics and gasoline service station workers in New Hampshire. Am J Ind Med. 1987;12(1):91-9.

52. Ragin C, Davis-Reyes B, Tadesse H, Daniels D, Bunker CH, Jackson M, Ferguson TS, Patrick AL, Tulloch-Reid MK, Taioli E. Farming, reported pesticide use, and prostate cancer. Am J Mens Health. 2013;7(2):102-9.

53. Depczynski J, Lower T. A review of prostate cancer incidence and mortality studies of farmers and non-farmers, 2002-2013. Cancer Epidemiol. 2014; 38(6):654-62.

54. Koutros S, Andreotti G, Berndt SI, Hughes Barry K, Lubin JH, Hoppin JA, Kamel F, Sandler DP, Burdette LA, Yuenger J, et al. Xenobiotic-metabolizing gene variants, pesticide use, and the risk of prostate cancer. Pharmacogenet Genomics. 2011;21(10):615-23.
55. Peters CE, Demers PA, Kalia S, Hystad P, Villeneuve PJ, Nicol A-M, Kreiger N, Koehoorn MW. Occupational exposure to solar ultraviolet radiation and the risk of prostate cancer. Occup Environ Med 2016;73(11):742-8.

56. Teschke K, Olshan AF, Daniels JL, De Roos AJ, Parks CG, Schulz M, Vaughan TL. Occupational exposure assessment in case-control studies: opportunities for improvement. Occup Environ Med. 2002;59(9):575-94.

57. Siemiatycki J, Day NE, Fabry J, Cooper JA. Discovering carcinogens in the occupational environment: a novel epidemiologic approach. J Natl Cancer Inst. 1981:66(2):217-25

\section{Submit your next manuscript to BioMed Central and we will help you at every step:}

- We accept pre-submission inquiries

- Our selector tool helps you to find the most relevant journal

- We provide round the clock customer support

- Convenient online submission

- Thorough peer review

- Inclusion in PubMed and all major indexing services

- Maximum visibility for your research

Submit your manuscript at www.biomedcentral.com/submit
C) Biomed Central 